\title{
UMA PROVA DA INSOLUBILIDADE DO \\ DÉCIMO PROBLEMA DE HILBERT \\ E RELAÇÕES COM \\ COMPLEXIDADE DE ALGORITMOS
}

Edson Tsukimoto

\author{
DISSERTAÇÃO APRESENTADA \\ AO \\ INSTITUTO DE MATEMÁTICA E ESTATÍSTICA \\ DA \\ UNIVERSIDADE DE SÃO PAULO \\ PARA \\ OBTENÇÃO DO GRAU \\ DE \\ MESTRE EM MATEMÁTICA
}

Área de Concentração: Lógica e Fundamentos

Orientador: Prof. Dr. Ricardo Bianconi

- São Paulo, agosto de 2000 - 


\title{
UMA PROVA DA INSOLUBILIDADE DO DÉCIMO PROBLEMA DE HILBERT E RELAÇÕES COM COMPLEXIDADE DE ALGORITMOS
}

\author{
Edson Tsukimoto
}

Este exemplar corresponde à redação final da dissertação apresentada por Edson Tsukimoto, devidamente corrigida e aprovada pela Comissão Julgadora

São Paulo, agosto de 2000

\section{Comissão Julgadora}

- Prof. Dr. Ricardo Bianconi - IME-USP

- Prof. Dr. Marcelo Finger

- Prof. Dr. Piotr Kozmider 


\section{Agradecimentos}

Ao orientador Ricardo Bianconi, pelo estímulo e tranqüilidade transmitidos durante este trabalho.

A meus pais, que tantos sacrifícios fizeram para que eu prosseguisse nos estudos.

À Olga, pela paciência e carinho que me deram forças para continuar.

Aos amigos do IME, Lúcia Ikemoto, João Paulo, Clóvis, Jefferson, Amélia, Rolo, Gaspar, Takeo, Solange, Edson, Sidney, Lenir, Samuel e em especial aos companheiros de batalha que vêm me acompanhando desde a graduação, Alexandre "Batman" Scalzitti e Flávio "Aleatório" Yamamoto.

Ao pessoal do café, Dalvina, Jovita e Maria, que me mantiveram acordado ao longo dos anos.

À Jane do CIC e suas honoráveis buchadas de bode.

A todo pessoal da biblioteca, Célia, Cristina, Lúcia, Márcia, Marina, Max, Renato, Sílvia e Vera, pelo convívio gostoso e bem-humorado.

Finalmente, ao Professor Jacob Zimbarg Sobrinho por me mostrar a filosofia e a beleza que se ocultam sob o árido manto da matemática. 


\section{Resumo}

Em seu Décimo Problema, Hilbert indaga se existe um procedimento efetivo que decida se uma dada equação diofantina admite solução. Neste trabalho vamos mostrar uma prova, finalizada por Yuri Matyasevic na década de setenta, de que tal procedimento efetivo não existe.

Ao final, mostraremos como esse resultado tem relações com a teoria de complexidade de algoritmos. Mais especificamente, veremos que se a demonstração da insolubilidade do Décimo Problema de Hilbert puder ser formalizada em um certo fragmento da aritmética de Peano, em um sentido que iremos precisar, então NP $=$ coNP.

\section{Abstract}

Hilbert, in his Tenth Problem, questioned whether there would exist an effective procedure which decided if a given diofantine equation has solution. In this work, we will present a proof, published in the seventies by Yuri Matyasevic which states that such an effective procedure does not exist.

We will also show that the above result has implications with the theory of complexity of algorithms. Precisely, we will see that if the proof of the unsolvability of the Hilbert's Tenth Problem can be stated in a certain fragment of the Peano's Arithmetic, in a certain sense we are going to make precise, then $\mathrm{NP}=\operatorname{coNP}$. 


\section{Conteúdo}

1 Definições básicas 1

1.1 O décimo problema de Hilbert . . . . . . . . . . . . . . . . . . . . . 1

1.2 Conjuntos diofantinos . . . . . . . . . . . . . . . . . . . . . . . . . . . . . . . 3

1.3 As funções $F(x, y), L(z)$ e $R(z) \ldots \ldots \ldots \ldots$. . . . . . . . . 4

1.4 A função $S(i, u) \ldots \ldots \ldots \ldots \ldots \ldots \ldots$

1.5 Esquema da prova . . . . . . . . . . . . . . . . . . . . . . . 9

2 Alguns lemas $\quad 11$

3 A função exponencial é diofantina $\quad 29$

$4 \quad$ As funções $\left(\begin{array}{l}n \\ k\end{array}\right), n ! \mathrm{e} \prod_{k=1}^{n}(a+b k)$ são diofantinas $\quad 37$

5 Quantificadores limitados são diofantinos $\quad 45$

6 Funções recursivas $\quad 53$

7 O décimo problema de Hilbert é insolúvel 58

8 Conjuntos recursivamente enumeráveis, grau e dimensão $\quad 66$

9 Relações com complexidade de algoritmos $\quad 70$

9.1 Definições básicas . . . . . . . . . . . . . . . . . . . . . . . . 71

9.1.1 Linguagens formais e máquinas de Turing . . . . . . . . . . . . . . . . . 71

9.1.2 Máquinas de Turing determinísticas (DTM) e a classe P . . . . . 71

9.1.3 Máquinas não-determinísticas e a classe NP . . . . . . . . . . . . . . 74

9.1.4 NP-completude . . . . . . . . . . . . . . . . . . . . . . . . . . . . 76

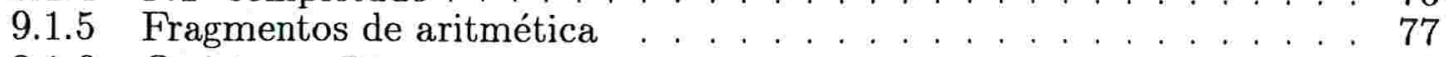

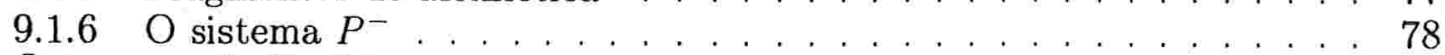

9.2 O teorema de Parikh $\ldots \ldots \ldots$

9.3 O teorema de Wilkie $\ldots \ldots \ldots$

$\begin{array}{ll}\text { Referências bibliográficas } & 99\end{array}$ 


\section{Capítulo 1}

\section{Definições básicas}

\subsection{O décimo problema de Hilbert}

O décimo problema de Hilbert pode ser assim formulado:

"Existe um algoritmo para decidir se dado um polinômio $P\left(x_{1}, \ldots, x_{n}\right)$ com coeficientes inteiros, existem ou não soluções inteiras para a equação $P\left(x_{1}, \ldots, x_{n}\right)=0$ ??"

Aqui, vamos indagar a respeito de soluções no conjunto $\mathbb{N}$. Isso não será uma limitação, já que a insolubilidade do problema que procura soluções em IN implica a insolubilidade do problema original.

De fato, suponhamos que $H$ seja um algoritmo que solucione o problema que busca

soluções em $\mathbb{Z}$. Dado então um polinômio $P\left(x_{1}, \ldots, x_{n}\right)$ com coeficientes inteiros, poderíamos decidir se existem $x_{1}, \ldots, x_{n} \in \mathbb{N}$ tal que

$$
P\left(x_{1}, \ldots, x_{n}\right)=0
$$


da seguinte maneira: tomamos o algoritmo $H$ para decidir se existem $a_{i}, b_{i}, c_{i}, d_{i} \in \mathbb{Z}$, $i=1, \ldots, n$, tal que

$$
P\left(a_{1}^{2}+b_{1}^{2}+c_{1}^{2}+d_{1}^{2}, \ldots, a_{n}^{2}+b_{n}^{2}+c_{n}^{2}+d_{n}^{2}\right)=0
$$

Teríamos então a seguinte situação:

- Se existe solução em $\mathbb{Z}$ para (1.2), então existe solução em $\mathbb{N}$ para (1.1), pois basta tomar $x_{i}=a_{i}^{2}+b_{i}^{2}+c_{i}^{2}+d_{i}^{2}$, para $i=1, \ldots, n$;

- Se não existe solução em $\mathbb{Z}$ para (1.2), então não existe solução em $\mathbb{N}$ para (1.1), pois se existisse $x_{1}, \ldots, x_{n} \in \mathbb{N}$ satisfazendo (1.1), então pelo teorema de Lagrange, que afirma que todo número natural pode ser escrito como a soma de quatro quadrados, existiriam $a_{i}, b_{i}, c_{i}, d_{i} \in \mathbb{Z}$, para $i=1, \ldots, n$ tais que $x_{i}=a_{i}^{2}+b_{i}^{2}+c_{i}^{2}+d_{i}^{2}$, e portanto $a_{i}, b_{i}, c_{i}, d_{i} \in \mathbb{Z}$ seria uma solução para a equação (1.2), contra nossa suposição.

Isso demonstra nossa afirmação.

Fazemos ainda mais uma pequena restrição por comodidade técnica: excluiremos o zero do domínio das funções.

Deste ponto em diante, salvo menção explícita, os polinômios terão seus coeficientes em $\mathbb{Z}$, e suas variáveis, assim como de outras funções, percorrerão o conjunto dos números inteiros positivos. 


\subsection{Conjuntos diofantinos}

Definiçäo 1.1 Um conjunto $S \subseteq \mathbb{N}_{*}^{n}$ é diofantino se e somente se existe um polinômio $P\left(x_{1}, \ldots, x_{n}, t_{1}, \ldots, t_{m}\right)$ tal que

$$
\left(x_{1}, \ldots, x_{n}\right) \in S \Leftrightarrow \exists t_{1}, \ldots, t_{m}\left(P\left(x_{1}, \ldots, x_{n}, t_{1}, \ldots, t_{m}\right)=0\right) .
$$

Definição 1.2 Um predicado $A\left(x_{1}, \ldots, x_{n}\right)$ é diofantino se e somente se o conjunto

$$
S=\left\{\left(x_{1}, \ldots, x_{n}\right): A\left(x_{1}, \ldots, x_{n}\right)\right\}
$$

é diofantino.

Definição 1.3 Uma função $f\left(x_{1}, \ldots, x_{n}\right)$ é diofantina se e somente se seu gráfico é diofantino, ou seja, se e somente se existe um polinômio $P\left(x_{1}, \ldots, x_{n}, y, t_{1}, \ldots, t_{m}\right)$ tal que

$$
y=f\left(x_{1}, \ldots, x_{n}\right) \Leftrightarrow \exists t_{1}, \ldots, t_{m}\left(P\left(x_{1}, \ldots, x_{n}, y, t_{1}, \ldots, t_{m}\right)=0\right) .
$$

Exemplos: Os predicados $x \leq y, x \mid y, x \equiv y(\bmod m)$ e as funções $q(x, y)$ e $r(x, y)$ (o quociente e o resto da divisão de $x$ por $y$, respectivamente) são diofantinos, pois

$$
\begin{aligned}
x \leq y & \Leftrightarrow \exists t(x+(t-1)-y=0) \\
x \mid y & \Leftrightarrow \exists t(x \cdot t-y=0) \\
x \equiv y(\bmod m) & \Leftrightarrow \exists t(x-m \cdot t-y=0) \\
q(x, y)=z & \Leftrightarrow \exists r(x=y \cdot z+r \wedge 0 \leq r<y) \\
r(x, y)=z & \Leftrightarrow \exists q(x=y \cdot q+z \wedge 0 \leq z<y) .
\end{aligned}
$$


Proposição 1.4 Se $A$ e $B$ são predicados diofantinos, então $\exists x A, A \vee B, A \wedge B$ também são predicados diofantinos.

Demonstração: Sejam $P$ e $Q$ polinômios tais que

$$
\begin{aligned}
& A\left(x_{1}, \ldots, x_{n}\right) \Leftrightarrow \exists t_{1}, \ldots, t_{m}\left(P\left(x_{1}, \ldots, x_{n}, t_{1}, \ldots, t_{m}\right)=0\right) \quad \mathrm{e} \\
& B\left(x_{1}, \ldots, x_{n}\right) \Leftrightarrow \exists t_{1}, \ldots, t_{m}\left(Q\left(x_{1}, \ldots, x_{n}, t_{1}, \ldots, t_{m}\right)=0\right) .
\end{aligned}
$$

Então

- $\exists x_{i} A\left(x_{1}, \ldots, x_{n}\right) \Leftrightarrow \exists x_{i}, t_{1}, \ldots, t_{m}\left(P\left(x_{1}, \ldots, x_{n}, t_{1}, \ldots, t_{m}\right)=0\right)$;

- $A\left(x_{1}, \ldots, x_{n}\right) \vee B\left(x_{1}, \ldots, x_{n}\right) \Leftrightarrow \exists y_{1}, \ldots, y_{m}, z_{1}, \ldots, z_{m}\left(P\left(x_{1}, \ldots, x_{n}, y_{1}, \ldots, y_{m}\right)\right.$ $\left.. Q\left(x_{1}, \ldots, x_{n}, z_{1}, \ldots, z_{m}\right)=0\right)$;

- $A\left(x_{1}, \ldots, x_{n}\right) \wedge B\left(x_{1}, \ldots, x_{n}\right) \Leftrightarrow \exists y_{1}, \ldots, y_{m}, z_{1}, \ldots, z_{m}\left(P^{2}\left(x_{1}, \ldots, x_{n}, y_{1}, \ldots, y_{m}\right)+\right.$ $\left.Q^{2}\left(x_{1}, \ldots, x_{n}, z_{1}, \ldots, z_{m}\right)=0\right)$.

Observação: Veremos adiante que os predicados diofantinos não são necessariamente fechados para $\neg, \rightarrow \mathrm{e} \forall$.

\subsection{As funções $F(x, y), L(z)$ e $R(z)$}

Essas funções farão uma bijeção entre os inteiros positivos e pares ordenados de inteiros positivos.

Consideremos a função $T(n)=\frac{n(n+1)}{2}, n \geq 0$. Definimos então a função $F(x, y)$ : 
Definição $1.5 \forall x, y \geq 1: F(x, y)=T(x+y-2)+y$

Proposição $1.6 \forall z \geq 1, \exists ! x, y \geq 1(z=F(x, y))$

Demonstração: Como a função $T(n)$ é estritamente crescente, então $\forall z \geq 1, \exists$ !n tal que

$$
T(n)<z \leq T(n+1)=\frac{(n+1)(n+2)}{2}=T(n)+n+1
$$

ou seja,

$$
\forall z \geq 1, \quad \exists ! n \geq 0, \exists ! y \geq 1(z=T(n)+y \wedge y \leq n+1)
$$

ou ainda,

$$
\forall z \geq 1, \quad \exists ! n \geq 0, \quad \exists ! x, y \geq 1(z=T(n)+y \wedge n=x+y-2)
$$

e portanto

$$
\forall z \geq 1, \exists ! x, y \geq 1(z=T(x+y-2)+y)
$$

Proposição 1.7 A função $F: \mathbb{N}_{*}^{2} \rightarrow \mathbb{N}_{*}$ é bijetora.

Demonstração: Segue da Proposição 1.6.

A Proposição 1.7 permite definir a função inversa $F^{-1}: \mathbb{N}_{*} \rightarrow \mathbb{N}_{*}^{2}$.

Sejam $L(z)$ e $R(z)$ as funções correspondentes à primeira e segunda coordenadas de $F^{-1}$, ou seja, $F^{-1}(z)=(L(z), R(z))$. Valem então as seguintes relações:

(a) $\forall z \geq 1, F\left(F^{-1}(z)\right)=F(L(z), R(z))=z$; 
(b) $\forall x, y \geq 1, F^{-1}(F(x, y))=(L(F(x, y)), R(F(x, y)))=(x, y)$ e portanto, $\forall x, y \geq 1$, $L(F(x, y))=x$ e $R(F(x, y))=y$.

Proposição $1.8 \forall x, y \geq 1: 1 \leq R(z) \leq z$ e $1 \leq L(z) \leq z$.

Demonstração: Segue da Proposição 1.6 e das definições de $L(z)$ e $R(z)$ que $\forall z \geq 1$, $z=T(L(z)+R(z)-2)+R(z), \operatorname{com} L(z), R(z) \geq 1$. É então imediato que $1 \leq R(z) \leq z$.

Para mostrar que $L(z) \leq z$, notemos primeiramente que $\forall n \geq 0, n \leq T(n)$. Portanto, $L(z)-1 \leq T(L(z)-1)$ e como $T$ é uma função estritamente crescente, temos então que

$$
L(z)-1 \leq T(L(z)-1) \leq T(L(z)+R(z)-2)<T(L(z)+R(z)-2)+R(z)=z .
$$

Portanto, $1 \leq L(z) \leq z$

Proposição 1.9 As funções $F(x, y), L(z)$ e $R(z)$ são diofantinas.

Demonstração: Pela Proposição 1.6,

$$
\forall z \geq 1, \exists ! x, y \geq 1(z=T(x+y-2)+y) .
$$

Lembrando que $T(n)=\frac{n(n+1)}{2}$, segue das definições das funções $F(x, y), L(z)$ e $R(z)$ que

$$
\begin{aligned}
z=F(x, y) & \Leftrightarrow 2 z=(x+y-2)(x+y-1)+2 y \\
x=L(z) & \Leftrightarrow \exists y(2 z=(x+y-2)(x+y-1)+2 y) \\
y=R(z) & \Leftrightarrow \exists x(2 z=(x+y-2)(x+y-1)+2 y)
\end{aligned}
$$

Portanto, $F(x, y), L(z)$ e $R(z)$ são funções diofantinas. 


\subsection{A função $S(i, u)$}

A função $S(i, u)$, uma pequena variação da função $\beta$ de Gödel, permitirá codificar seqüências de números naturais.

Definição $1.10 \forall i, u \geq 1, \quad S(i, u)=r(L(u), 1+i . R(u)$ ) (resto da divisão de $L(u)$ por $1+i \cdot R(u))$.

Proposição $1.11 \forall i, u \geq 1,0 \leq S(i, u) \leq u$.

Demonstração: Como $S(i, u)$ é o resto da divisão de $L(u)$ por $1+i \cdot R(u)$, então existe um único número $q$ tal que

$$
L(u)=q \cdot(1+i R(u))+S(i, u)
$$

$\operatorname{com} 0 \leq S(i, u)<1+i \cdot R(u)$

Então $0 \leq S(i, u) \leq L(u)$ e, pela Proposição $1.8, L(u) \leq u$. Portanto, $0 \leq S(i, u) \leq u$.

Proposição 1.12 A função $S(i, u)$ é diofantina.

Demonstração: A função $r(x, y)$, o resto da divisão de $x$ por $y$, é diofantina, pois

$$
r(x, y)=z \Leftrightarrow \exists q(x=y \cdot q+z \wedge 0 \leq z<y)
$$

Então, $S(i, u)$ é uma função diofantina, pois

$$
S(i, u)=z \Leftrightarrow \exists x, y(r(x, y)=z \wedge x=L(u) \wedge y=1+i . R(u))
$$


e $x=L(u)$ e $y=1+i \cdot R(u)$ são diofantinas pela Proposição 1.9.

Proposição 1.13 Para toda seqüência de números naturais $a_{1}, a_{2}, \ldots, a_{n}$, existe um número u tal que para cada $i=1, \ldots, n, S(i, u)=a_{i}$.

Demonstração: Seja $a_{1}, a_{2}, \ldots, a_{n}$ uma dada seqüência de números naturais,

$$
m=\max \left\{n, a_{1}, a_{2}, \ldots, a_{n}\right\} \text { e } y=m !
$$

Afirmação: $0 \leq i<j \leq n \rightarrow \operatorname{mdc}(1+i . y, 1+j \cdot y)=1$.

De fato, seja $0 \leq i<j \leq n$ e suponhamos que $\operatorname{mdc}(1+i . y, 1+j . y)>1$.

Seja então $p$ um primo tal que $p \mid 1+i . y$ e $p \mid 1+j . y$.

Se $p \mid y$, como $p \mid 1+i y$, então teríamos que $p \mid 1$. Portanto, $p \nmid y$.

Por outro lado, se $p \mid 1+i . y$ e $p \mid 1+j . y$, então $p \mid(j-i) . y$, e como $p$ é um primo tal que $p \nmid y$, então $p \mid j-i$. Como $j-i>0$, então $p \leq j-i$, e como $0<j-i \leq n \leq m=$ $\max \left\{n, a_{1}, a_{2}, \ldots, a_{n}\right\}$, então $p \leq m$, e portanto $p \mid m$ !, isto é , $p \mid y$, uma contradição.

Podemos agora aplicar o teorema chinês do resto para encontrar uma solução para o sistema $x \equiv a_{i}(\bmod 1+i . y), i=1, \ldots, n$. Então $r(x, 1+i . y)=r\left(a_{i}, 1+i . y\right), i=1, \ldots, n$. Mas $a_{i}<1+i . y$, pois $y=m !$ e $m=\max \left\{n, a_{1}, a_{2}, \ldots, a_{n}\right\}$. Logo, $r\left(a_{i}, 1+i . y\right)=a_{i}$, e portanto, para $i=1, \ldots, n, r(x, 1+i y)=a_{i}$.

Seja agora $u=F(x, y)$. Então $L(u)=x$ e $R(u)=y$. 
Portanto, para $i=1, \ldots, n$, teremos que

$$
S(i, u)=r(L(u), 1+i \cdot R(u))=r(x, 1+i . y)=a_{i} .
$$

\subsection{Esquema da prova}

A prova da insolubilidade do décimo problema de Hilbert é obtida por meio de várias reduções. Graças aos trabalhos de Júlia Robinson [11], era sabido que a insolubidade do décimo problema de Hilbert se seguiria se a função exponencial fosse diofantina, fato este provado por Matyasevic em 1970 [8], [9], encerrando assim a questão levantada por Hilbert em seu décimo problema.

Nos capítulos que se seguem, definiremos duas funções $x_{n}(a)$ e $y_{n}(a), a>1$ fixo, por

$$
x_{n}(a)+y_{n}(a) \sqrt{a^{2}-1} \stackrel{\text { def. }}{=}\left(a+\sqrt{a^{2}-1}\right)^{n},
$$

e mostraremos que $x_{n}(a)$ e $y_{n}(a)$ são diofantinas. Desse fato, com o auxílio da equação de Pell, resultará que a função exponencial é diofantina. A seguir, mostramos que as funções $f(n, k)=\left(\begin{array}{l}n \\ k\end{array}\right), g(n)=n !$ e $h(a, b, n)=\prod_{k=1}^{n}(a+b k)$ também são diofantinas, o que permitirá mostrar que predicados diofantinos são fechados para os quantificadores limitados $\forall x \leq t$ e $\exists y \leq t$. Com o auxílio desses resultados, chegamos finalmente à insolubilidade do décimo problema de Hilbert, segundo o esquema que apresentamos a seguir. 


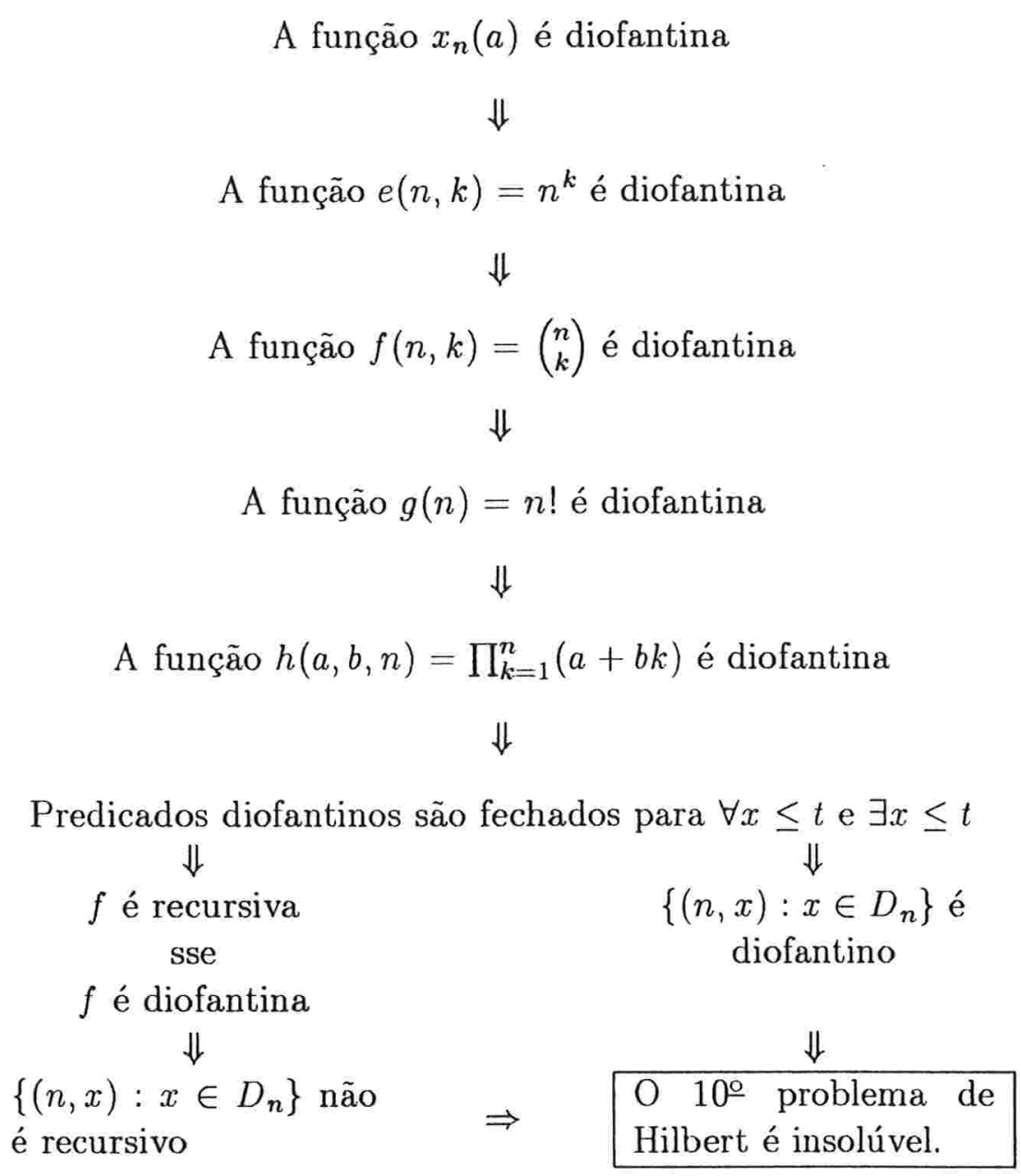




\section{Capítulo 2}

\section{Alguns lemas}

Um resultado chave na demonstração da insolubilidade do décimo problema de Hilbert consiste na prova de que a função exponencial é diofantina [8]. Para esse fim, apresentaremos agora alguns lemas relacionados com a equação de Pell.

Seja $a>1$ e $d=a^{2}-1$.

Equação de Pell: $\left\{\begin{array}{l}x^{2}-d y^{2}=1 \\ x, y \geq 0\end{array}\right.$

Se $x, y$ satisfazem a equação de Pell para $a>1$ fixado, indicaremos esse fato por $\operatorname{Pell}_{a}(x, y)$. Se estiver claro no contexto o número $a>1$ fixado, omitiremos o índice $a \mathrm{e}$ escreveremos simplesmente $\operatorname{Pell}(x, y)$.

É importante notar que $\sqrt{d}$ não é um número inteiro, pois se $d=a^{2}-1=b^{2}$, então $b<a, \operatorname{logo} b+1 \leq a$, e portanto $(b+1)^{2} \leq a^{2}$, ou seja, $(b+1)^{2}=b^{2}+1+2 b=a^{2}+2 b \leq a^{2}$, de onde obteríamos $b=0, \operatorname{mas} b \neq 0$, pois supomos $b^{2}=d=a^{2}-1>0$, já que $a>1$. 
Para os lemas que se seguem, salvo menção explícita, estarão fixados $a>1$ e $d=a^{2}-1$.

Lema 2.1 Nâo existem números $x, y$ tais que $\operatorname{Pell}(x, y)$ e $1<x+y \sqrt{d}<a+\sqrt{d}$.

Demonstração: Como $d=a^{2}-1$, então $(a+\sqrt{d})(a-\sqrt{d})=1$ e como $\operatorname{Pell}(x, y)$, então $x>0$ e $y>0$ e também $(x+y \sqrt{d})(x-y \sqrt{d})=x^{2}-d y^{2}=1$. Portanto,

$$
1=(a+\sqrt{d})(a-\sqrt{d})=(x+y \sqrt{d})(x-y \sqrt{d})
$$

Como $a+\sqrt{d}>0$ e $x+y \sqrt{d}>0$, então $(a-\sqrt{d})>0$ e $(x-y \sqrt{d})>0$.

Vamos supor agora que

$$
1<x+y \sqrt{d}<a+\sqrt{d}
$$

De (2.1) e (2.2), temos que $a-\sqrt{d}<x-y \sqrt{d}$ e como supomos $x+y \sqrt{d}>1$, de (2.1) teremos $x-y \sqrt{d}<1$, logo $a-\sqrt{d}<x-y \sqrt{d}<1$ e portanto

$$
-1<-x+y \sqrt{d}<-a+\sqrt{d}
$$

Somando (2.2) e (2.3), obtemos

$$
0<2 y \sqrt{d}<2 \sqrt{d}
$$

e portanto $0<y<1$. Absurdo, pois $y \in \mathbb{N}^{*}$.

Lema 2.2 Sejam $x, y, z, w$ tais que $\operatorname{Pell}(x, y)$, Pell $(z, w)$ e $u, v$ tais que

$$
u+v \sqrt{d}=(x \pm y \sqrt{d})(z \pm w \sqrt{d})
$$

então $\operatorname{Pell}(u, v)$ 
Demonstração: Vamos mostrar o caso $u+v \sqrt{d}=(x+y \sqrt{d})(z+w \sqrt{d})$ :

$$
\begin{aligned}
u+v \sqrt{d} & =(x+y \sqrt{d})(z+w \sqrt{d}) \\
& =(x z+y w d)+(x w+y z) \sqrt{d}
\end{aligned}
$$

Então, $u=x z+y w d$ e $v=x w+y z$. Logo,

$$
\begin{aligned}
u^{2} & =(x z+y w d)^{2}=x^{2} z^{2}+2 x y z w d+y^{2} w^{2} d^{2} \\
d v^{2} & =d(x w+y z)^{2}=x^{2} w^{2} d+2 x y z w d+y^{2} z^{2} d
\end{aligned}
$$

Então temos que

$$
\begin{aligned}
u^{2}-d v^{2} & =x^{2} z^{2}+y^{2} w^{2} d^{2}-x^{2} w^{2} d-y^{2} z^{2} d \\
& =x^{2}\left(z^{2}-d w^{2}\right)-d y^{2}\left(z^{2}-d w^{2}\right)
\end{aligned}
$$

e como $\operatorname{Pell}(z, w)$, então $z^{2}-d w^{2}=1, \operatorname{logo}$

$$
u^{2}-d v^{2}=x^{2}-d y^{2}
$$

e como Pell $(x, y)$, então $x^{2}-d y^{2}=1, \operatorname{logo}$

$$
u^{2}-d v^{2}=1
$$

Portanto, $\operatorname{Pell}(u, v)$.

As demonstrações para os outros casos são feitas de forma análoga.

Definição 2.3 Dado $a>1$, vamos definir duas funções $x_{n}(a)$ e $y_{n}(a)$ para $n \geq 0$ :

$$
x_{n}(a)+y_{n}(a) \sqrt{d} \stackrel{\text { def }}{=}(a+\sqrt{d})^{n} .
$$


Observação: Quando o contexto permitir, omitiremos a dependência de $a$ e escreveremos simplesmente $x_{n}, y_{n}$.

Lema 2.4 Para todo $n \geq 0$, Pell $\left(x_{n}, y_{n}\right)$.

Demonstração: Por indução sobre $n$ : para $n=0$ é imediato que Pell $\left(x_{0}, y_{0}\right)$, pois $x_{0}=1$ e $y_{0}=0$. Suponhamos $\operatorname{Pell}\left(x_{n}, y_{n}\right)$. Então

$$
\begin{aligned}
x_{n+1}+y_{n+1} \sqrt{d} & =(a+\sqrt{d})^{n+1}=(a+\sqrt{d})^{n}(a+\sqrt{d}) \\
& =\left(x_{n}+y_{n} \sqrt{d}\right)(a+\sqrt{d})
\end{aligned}
$$

É imediato que Pell $(a, 1)$ e, por hipótese de indução, Pell $\left(x_{n}, y_{n}\right)$, e portanto, a equação acima juntamente com o Lema 2.2 nos mostra que Pell $\left(x_{n+1}, y_{n+1}\right)$.

Portanto, $\forall n \geq 0$, Pell $\left(x_{n}, y_{n}\right)$.

Lema 2.5 Sejam $x, y \geq 0$ tais que Pell $(x, y)$. Então existe $n$ tal que $x=x_{n}$ e $y=y_{n}$.

Demonstração: Sejam $x, y \geq 0$ tais que $\operatorname{Pell}(x, y)$ e consideremos o número $x+y \sqrt{d}$. Notemos que $x+y \sqrt{d}>0$, pois $\operatorname{Pell}(x, y)$. Como a função $f(n)=(a+\sqrt{d})^{n}$ é estritamente crescente, existe $n \geq 0$ tal que

$$
(a+\sqrt{d})^{n} \leq x+y \sqrt{d}<(a+\sqrt{d})^{n+1} .
$$

Lembrando que $(a+\sqrt{d})^{n}=x_{n}+y_{n} \sqrt{d}$, temos que se $(a+\sqrt{d})^{n}=x+y \sqrt{d}$, então $x=x_{n}$ e $y=y_{n}$. Suponhamos agora que $(a+\sqrt{d})^{n}<x+y \sqrt{d}$. Então

$$
x_{n}+y_{n} \sqrt{d}=(a+\sqrt{d})^{n}<x+y \sqrt{d}<(a+\sqrt{d})^{n+1} .
$$




$$
=(a+\sqrt{d})^{n}(a+\sqrt{d})=\left(x_{n}+y_{n} \sqrt{d}\right)(a+\sqrt{d}) .
$$

Por outro lado, como $\left(x_{n}+y_{n} \sqrt{d}\right)\left(x_{n}-y_{n} \sqrt{d}\right)=1$, então $x_{n}-y_{n} \sqrt{d}>0$.

Segue das inequações acima que

$$
\begin{aligned}
\left(x_{n}+y_{n} \sqrt{d}\right)\left(x_{n}-y_{n} \sqrt{d}\right) & <(x+y \sqrt{d})\left(x_{n}-y_{n} \sqrt{d}\right) \\
& <\left(x_{n}+y_{n} \sqrt{d}\right)\left(x_{n}-y_{n} \sqrt{d}\right)(a+\sqrt{d})
\end{aligned}
$$

ou seja, $1<(x+y \sqrt{d})\left(x_{n}-y_{n} \sqrt{d}\right)<a+\sqrt{d}$.

Como por hipótese Pell $(x, y)$ e pelo Lema 2.4, Pell $\left(x_{n}, y_{n}\right)$, segue do Lema 2.2 que $(x+y \sqrt{d})\left(x_{n}-y_{n} \sqrt{d}\right)$ é uma solução da equação de Pell.

Portanto, o Lema 2.1 contradiz a inequação

$$
1<(x+y \sqrt{d})\left(x_{n}-y_{n} \sqrt{d}\right)<a+\sqrt{d} .
$$

Portanto, existe $n \geq 0$ tal que $x=x_{n}$ e $y=y_{n}$.

\section{Lema 2.6}

(a) $x_{m+n}=x_{m} x_{n}+d y_{m} y_{n}$

$$
y_{m+n}=x_{n} y_{m}+x_{m} y_{n}
$$

(b) Se $n \leq m$ :

$$
\begin{aligned}
& x_{m-n}=x_{m} x_{n}-d y_{m} y_{n} \\
& y_{m-n}=x_{m} y_{m}-x_{m} y_{n}
\end{aligned}
$$


Demonstração: (a) $x_{m+n}+y_{m+n} \sqrt{d}=(a+\sqrt{d})^{m+n}=(a+\sqrt{d})^{m}(a+\sqrt{d})^{n}=$ $\left(x_{m}+y_{n} \sqrt{d}\right)\left(x_{n}+y_{n} \sqrt{d}\right)=\left(x_{m} x_{n}+d y_{m} y_{n}\right)+\left(x_{n} y_{m}+x_{m} y_{n}\right) \sqrt{d}$. Portanto,

$$
x_{m+n}=x_{m} x_{n}+d y_{m} y_{n}
$$

e

$$
y_{m+n}=x_{n} y_{m}+x_{m} y_{n}
$$

(b) Notemos que $\left(x_{m-n}+y_{m-n} \sqrt{d}\right)\left(x_{n}+y_{n} \sqrt{d}\right)=\left(x_{m-n} x_{n}+y_{m-n} y_{n} d\right)+$ $\left(x_{m-n} y_{n}+x_{n} y_{m-n}\right) \sqrt{d}$ e pela parte (a) deste lema:

$$
x_{m-n} x_{n}+y_{m-n} y_{n} d=x_{(m-n)+n}=x_{m}
$$

e

$$
x_{m-n} y_{n}+x_{n} y_{m-n}=y_{(m-n)+n}=y_{m}
$$

Portanto,

$$
\left(x_{m-n}+y_{m-n} \sqrt{d}\right)\left(x_{n}+y_{n} \sqrt{d}\right)=x_{m}+y_{m} \sqrt{d} .
$$

Logo

$$
\begin{aligned}
x_{m-n}+y_{m-n} \sqrt{d} & =\left(x_{m}+y_{m} \sqrt{d}\right)\left(x_{n}-y_{n} \sqrt{d}\right) \\
& =\left(x_{m} x_{n}-y_{m} y_{n} d\right)=\left(x_{n} y_{m}-x_{m} y_{n} \sqrt{d}\right) .
\end{aligned}
$$

Portanto,

$$
x_{m-n}=x_{m} x_{n}-y_{m} y_{n} d \quad \text { e } \quad y_{m-n}=x_{n} y_{m}-x_{m} y_{n}
$$

Lema $2.7 x_{m \pm 1}=a x_{m} \pm d y_{m} \quad e \quad y_{m \pm 1}=a y_{m} \pm x_{m}$. 
Demonstração: Basta tomar $n=1$ no Lema 2.6.

Lema $2.8 \operatorname{mdc}\left(x_{n}, y_{n}\right)=1$

Demonstração: Suponha $k \mid x_{n}$ e $k \mid y_{n}$. Então $k \mid x_{n}^{2}-d y_{n}^{2}$, mas $x_{n}^{2}-d y_{n}^{2}=1, \operatorname{logo} k=1$. Portanto, $\operatorname{mdc}\left(x_{n}, y_{n}\right)=1$.

Lema $2.9 y_{n} \mid y_{n k}$.

Demonstração: Por indução sobre $k$ : Para $k=0$ é imediato. Agora, pelo Lema 2.6: $y_{n(k+1)}=x_{n} y_{n k}+x_{n k} y_{n}$. Como por hipótese de indução $y_{n} \mid y_{n k}$, a expressão acima mostra que $y_{n} \mid y_{n(k+1)}$. Portanto, $y_{n} \mid y_{n k}$.

Lema $2.10 n\left|t \Leftrightarrow y_{n}\right| y_{t}$.

Demonstração: $(\Rightarrow)$ Segue do Lema 2.9

$(\Leftarrow)$ Suponha que $y_{n} \mid y_{t}$ mas $n \dagger t$ e seja $t=n q+r$, com $0<r<n$. Então, usando o Lema 2.6:

$$
y_{t}=y_{n q+r}=x_{r} y_{n q}+x_{n q} y_{r}
$$

Pelo Lema 2.9, $y_{n} \mid y_{n q}$, logo, como $y_{n} \mid y_{t}$ e $y_{n} \mid x_{r} y_{n q}$, temos que $y_{n} \mid x_{n . q} y_{r}$. Afirmação: $\operatorname{mdc}\left(y_{n}, x_{n q}\right)=1$. 
De fato, se $k \mid y_{n}$ e $k \mid x_{n q}$, então pelo Lema 2.9, $k \mid y_{n q}$. Mas pelo Lema 2.8,

$$
\operatorname{mdc}\left(x_{n q}, y_{n q}\right)=1
$$

$\operatorname{logo}, k=1$. Agora, como $y_{n} \mid x_{n q} y_{r}$ e $\operatorname{mdc}\left(y_{n}, x_{n q}\right)=1$, temos que $y_{n} \mid y_{r}$. Além disso, como $r \neq 0$, então $y_{r} \neq y_{0}=0$. Portanto,

$$
y_{n} \leq y_{r}
$$

Por outro lado, pelo Lema 2.7, a função $y_{n}$ é estritamente crescente e como $r<n$, temos que $y_{r}<y_{n}$, contra (2.4). Portanto, $r=0$ e $t=n q$, isto é, $n \mid t$.

Lema $2.11 y_{n k} \equiv k x_{n}^{k-1} y_{n}\left(\bmod y_{n}^{3}\right)$

\section{Demonstração:}

$$
\begin{aligned}
& x_{n k}+y_{n k} \sqrt{d}=(a+\sqrt{d})^{n k} \\
& =\left(x_{n}+y_{n} \sqrt{d}\right)^{k}=\sum_{j=0}^{k}\left(\begin{array}{l}
k \\
j
\end{array}\right) x_{n}^{k-j} y_{n}^{j} \sqrt{d}^{j} \\
& =\sum_{\substack{j=0 \\
j \text { par }}}^{k}\left(\begin{array}{l}
k \\
j
\end{array}\right) x_{n}^{k-j} y_{n}^{j} d^{j / 2}+\left(\sum_{\substack{j=1 \\
j \text { impar }}}^{k}\left(\begin{array}{l}
k \\
j
\end{array}\right) x_{n}^{k-j} y_{n}^{j} d^{(j-1) / 2}\right) \sqrt{d}
\end{aligned}
$$

Portanto,

$$
\begin{aligned}
y_{n k} & =\sum_{\substack{j=1 \\
j \text { impar }}}^{k}\left(\begin{array}{l}
k \\
j
\end{array}\right) x_{n}^{k-j} y_{n}^{j} d^{(j-1) / 2} \\
& =k x_{n}^{k-1} y_{n}+y_{n}^{3} \sum_{\substack{j=3 \\
j \text { impar }}}^{k}\left(\begin{array}{l}
k \\
j
\end{array}\right) x_{n}^{k-j} y_{n}^{j-3} d^{(j-1) / 2}
\end{aligned}
$$

e essa expressão nos mostra que

$$
y_{n k} \equiv k x_{n}^{k-1} y_{n}\left(\bmod y_{n}^{3}\right)
$$


Lema $2.12 y_{n}^{2} \mid y_{n y_{n}}$.

Demonstração: Fazendo $k=y_{n}$ no Lema 2.11, obtemos

$$
y_{n y_{n}} \equiv y_{n} x^{y_{n}-1} y_{n}=x_{n}^{y_{n}-1} y_{n}^{2}\left(\bmod y_{n}^{3}\right)
$$

ou seja, $y_{n}^{3} \mid y_{n \cdot y_{n}}-x_{n}^{y_{n}-1} y_{n}^{2}$. Portanto, $y_{n}^{2} \mid y_{n y_{n}}$.

Lema $2.13 S e y_{n}^{2} \mid y_{t}$, então $y_{n} \mid t$.

Demonstração: Como $y_{n}^{2} \mid y_{t}$, então $y_{n} \mid y_{t}$, e pelo Lema 2.10, $n \mid t$. Seja $t=k n$. Pelo Lema 2.11, $y_{n k} \equiv k x_{n}^{k-1} y_{n}\left(\bmod y_{n}^{3}\right)$, isto é, $y_{n}^{3} \mid y_{n k}-k x_{n}^{k-1} y_{n}$. Como por hipótese $y_{n}^{2} \mid y_{t}=$ $y_{n k}$, a expressão acima mostra que $y_{n}^{2} \mid k x_{n}^{k-1} y_{n}$, ou ainda, que $y_{n} \mid k x_{n}^{k-1}$. Pelo Lema 2.8, $\operatorname{mdc}\left(x_{n}, y_{n}\right)=1$, então $y_{n} \mid k$, e lembrando que $t=k n$, temos que $y_{n} \mid t$.

Lema $2.14 x_{n+1}=2 a x_{n}-x_{n-1} \quad e \quad y_{n+1}=2 a y_{n}-y_{n-1}$.

Demonstração: Pelo Lema 2.7, temos que

$$
\begin{array}{llc}
x_{n+1}=a x_{n}+d y_{n} \quad \text { e } & x_{n-1}=a x_{n}-d y_{n} \\
y_{n+1}=a y_{n}+x_{n} & \text { e } & y_{n-1}=a y_{n}-x_{n},
\end{array}
$$

de onde obtemos

$$
x_{n+1}+x_{n-1}=2 a x_{n} \quad \text { e } \quad y_{n+1}+y_{n-1}=2 a y_{n}
$$

Portanto, $x_{n+1}=2 a x_{n}-x_{n-1}$ e $y_{n+1}=2 a y_{n}-y_{n-1}$. 
Lema $2.15 y_{n} \equiv n(\bmod a-1)$.

Demonstração: Por indução sobre $n$ : o resultado é imediato para $n=0,1$. Por hipótese de indução, temos que $y_{n-1} \equiv n-1(\bmod a-1)$ e $y_{n} \equiv n(\bmod a-1)$. Como $a \equiv 1(\bmod a-1)$, temos então que $2 a y_{n} \equiv 2 n(\bmod a-1)$. Agora, pelo Lema 2.14 , $y_{n+1}=2 a y_{n}-y_{n-1}$. Então, $y_{n+1}=2 a y_{n}-y_{n-1} \equiv 2 n-(n-1)=n+1(\bmod a-1)$. Portanto, $y_{n} \equiv n(\bmod a-1)$.

Lema 2.16 Se $a \equiv b(\bmod c)$, então para todo $n$ :

$$
x_{n}(a) \equiv x_{n}(b)(\bmod c) \quad e \quad y_{n}(a) \equiv y_{n}(b)(\bmod c)
$$

Demonstração: Vamos mostrar que $x_{n}(a) \equiv x_{n}(b)(\bmod c)$ por indução sobre $n$ :

O resultado vale para $n=0,1$, pois $x_{0}(a)=x_{0}(b)=1$ e $x_{1}(a)=a$ e $x_{1}(b)=b$. Como $a \equiv b(\bmod c)$ e por hipótese de indução $x_{n}(a) \equiv x_{n}(b)(\bmod c)$, então

$$
2 a x_{n}(a) \equiv 2 b x_{n}(b)(\bmod c)
$$

Agora, pelo Lema 2.14, $x_{n+1}=2 a x_{n}-x_{n-1}$, então

$$
x_{n+1}(a)=2 a x_{n}(a)-x_{n-1}(a) \equiv 2 b x_{n}(b)-x_{n-1}(b)=x_{n+1}(b)(\bmod c) .
$$

A demonstração para o outro caso é análoga.

Lema $2.17 n$ é par se e somente se $y_{n}$ é par. 
Demonstração: Pelo Lema 2.14, $y_{k+1}=2 a y_{k}-y_{k-1}$. Então,

$$
y_{k+1}=2 a y_{k}-y_{k-1} \equiv y_{k-1}(\bmod 2)
$$

e a congruência $y_{k+1} \equiv y_{k-1}(\bmod 2) \operatorname{mostra}$ que se $n$ é par, $y_{n} \equiv y_{0}=0(\bmod 2), \operatorname{logo}$, $y_{n}$ é par. Se $n$ é ímpar, $y_{n} \equiv y_{1}=1(\bmod 2)$, logo $y_{n}$ é ímpar.

Lema $2.18 x_{n}-y_{n}(a-y) \equiv y^{n}\left(\bmod 2 a y-y^{2}-1\right)$

Demonstração: Por indução sobre $n$ : o resultado vale para $n=0,1$, pois $x_{0}-y_{0}(a-y)=$ $1=y^{0}$ e $x_{1}-y_{1}(a-y)=y=y^{1}$. Pelo Lema $2.14, x_{n+1}=2 a x_{n}-x_{n-1}$ e $y_{n+1}=2 a y_{n}-y_{n-1}$. Então $x_{n+1}-y_{n+1}(a-y)=\left(2 a x_{n}-x_{n-1}\right)-\left(2 a y_{n}-y_{n-1}\right)(a-y)=2 a x_{n}-x_{n-1}-2 a^{2} y_{n}+$ $2 a y_{n} y+a y_{n-1}-y y_{n-1}=2 a\left(x_{n}-y_{n}(a-y)\right)-\left(x_{n-1}-y_{n-1}(a-y)\right)$.

Por hipótese de indução, da expressão acima, obtemos

$$
x_{n+1}-y_{n+1}(a-y) \equiv 2 a y^{n}-y^{n-1}=y^{n-1}(2 a y-1)\left(\bmod 2 a y-y^{2}-1\right) .
$$

Notando que $2 a y-1 \equiv y^{2}\left(\bmod 2 a y-y^{2}-1\right)$, obtemos

$$
x_{n+1}-y_{n+1}(a-y) \equiv y^{n-1} y^{2}=y^{n+1}\left(\bmod 2 a y-y^{2}-1\right)
$$

Lema 2.19 Para todo $n, y_{n+1}>y_{n} \geq n$.

Demonstração: Pelo Lema 2.7, $y_{n+1}=x_{n}+a y_{n}$. Então, $y_{n+1}>y_{n}$.

Mostremos agora que $y_{n} \geq n$ por indução sobre $n$. É claro que $0 \leq y_{0}=0$. E pelo Lema 2.7, $y_{n+1}=a y_{n}+x_{n} \geq a_{n}+x_{n} \geq n+x_{n} \geq n+1$. 
Lema 2.20 Para todo $n, x_{n+1}>x_{n} \geq a^{n}$ e $x_{n} \leq(2 a)^{n}$.

Demonstração: Pelo Lema 2.7, $x_{n+1}=a x_{n}+d y_{n}$. Logo, $x_{n+1}=a x_{n}+d y_{n} \geq a x_{n}>x_{n}$.

Vamos mostrar $a^{n} \leq x_{n}$ por indução sobre $n: a^{0}=1 \leq 1=x_{0}$.

Agora, pelo Lema 2.7, $x_{n+1}=a x_{n}+d y_{n}$, logo, $a x_{n} \leq x_{n+1}$. Então, $a^{n+1}=a^{n} a \leq$ $a x_{n} \leq x_{n+1}$. Portanto, $a^{n} \leq x_{n}<x_{n+1}$.

Mostremos agora que $x_{n} \leq(2 a)^{n}$ por indução sobre $n$ : é claro que $x_{0}=1 \leq 1=(2 a)^{0}$.

Pelo Lema 2.14, $x_{n+1}=2 a x_{n}-x_{n-1}$, $\operatorname{logo} x_{n+1} \leq 2 a x_{n}$. Então, $x_{n+1} \leq 2 a x_{n} \leq$ $2 a(2 a)^{n}=(2 a)^{n+1}$. Portanto, $x_{n} \leq(2 a)^{n}$.

\section{Lema 2.21}
(a) $x_{2 n+j} \equiv-x_{j}\left(\bmod x_{n}\right)$;
(b) $S e 0 \leq j \leq 2 n$, então $x_{2 n-j} \equiv-x_{j}\left(\bmod x_{n}\right)$.

Demonstração: Usando o Lema 2.6, obtemos:

$$
\begin{aligned}
x_{2 n+j} & =x_{n+(n+j)}=x_{n} x_{n+j}+d y_{n} y_{n+j} \\
& =x_{n} x_{n+j}+d y_{n}\left(x_{j} y_{n}+x_{n} y_{j}\right)=x_{n} x_{n+j}+d y_{n}^{2} x_{j}+d y_{n} x_{n} y_{j} \\
& \equiv d y_{n}^{2} x_{j}\left(\bmod x_{n}\right)
\end{aligned}
$$

e como $d y_{n}^{2}=x_{n}^{2}-1$, temos que

$$
d y_{n}^{2} x_{j}=\left(x_{n}^{2}-1\right) x_{j}=x_{n}^{2} x_{j}-x_{j} \equiv-x_{j}\left(\bmod x_{n}\right) .
$$


Portanto, $x_{2 n+j} \equiv-x_{j}\left(\bmod x_{n}\right)$.

(b) Caso 1: $0 \leq j \leq n$.

Então, pelo Lema 2.6, da mesma forma que no item (a), teremos

$$
x_{2 n-j} \equiv-x_{j}\left(\bmod x_{n}\right)
$$

Caso 2: $n<j \leq 2 n$.

Seja $k=2 n-j$. Então $0 \leq k<n$, e portanto pelo caso 1 , temos que

$$
x_{2 n-k} \equiv-x_{k}\left(\bmod x_{n}\right)
$$

Mas $x_{2 n-k}=x_{2 n-(2 n-j)}=x_{j}$ e $x_{k}=x_{2 n-j}$. Portanto, $x_{2 n-j} \equiv-x_{j}\left(\bmod x_{n}\right)$.

\section{Lema 2.22}

$$
\begin{aligned}
& \text { (a) } \quad x_{4 n+j} \equiv x_{j}\left(\bmod x_{n}\right) \\
& \text { (b) } \quad \text { Se } 0 \leq j \leq 4 n \text {, entäo } x_{4 n-j} \equiv x_{j}\left(\bmod x_{n}\right) \text {. }
\end{aligned}
$$

Demonstração: (a) Usando duas vezes o Lema 2.21 a, temos

$$
x_{4 n+j}=x_{2 n+(2 n+j)} \equiv-x_{2 n+j} \equiv x_{j}\left(\bmod x_{n}\right) .
$$

(b) Caso 1: $0 \leq j \leq 2 n$.

Usando o Lema $2.21 \mathrm{a}$ e b, temos

$$
x_{4 n-j}=x_{2 n+(2 n-j)} \equiv-x_{2 n-j} \equiv x_{j}\left(\bmod x_{n}\right) .
$$


Caso 2: $2 n<j \leq 4 n$.

Seja $k=4_{n-j}$. Então $0 \leq k<2 n$. Pelo caso 1 , temos $x_{4 n-k} \equiv x_{k}\left(\bmod x_{n}\right)$. Mas $x_{4 n-k}=x_{4 n-(4 n-j)}=x_{j}$, e $x_{k}=x_{4 n-j}$. Portanto, $x_{4 n-j} \equiv x_{j}\left(\bmod x_{n}\right)$.

Lema 2.23 Seja $n>0,0 \leq i \leq j \leq 2 n$ e $x_{i} \equiv x_{j}\left(\bmod x_{n}\right)$. Então $(i=j)$ ou $(a=2 \wedge n=1 \wedge i=0 \wedge j=2)$.

Demonstração: Vamos necessitar dos seguintes fatos:

(1) $1=x_{0}<x_{1}<\cdots<x_{n-1}$ (pelo Lema 2.20);

(2) $2 x_{n-1} \leq x_{n}$,

pois pelo Lema $2.7, x_{n}=a x_{n-1}+d y_{n-1}, \operatorname{logo}, a x_{n-1} \leq x_{n}$, e como $a \geq 2$, temos que $2 x_{n-1} \leq a x_{n-1} \leq x_{n}$.

(3) $x_{n+1} \equiv-x_{n-1}\left(\bmod x_{n}\right)$

$x_{n+2} \equiv-x_{n-2}\left(\bmod x_{n}\right)$

$\vdots$

$x_{2 n} \equiv-x_{0}\left(\bmod x_{n}\right)$

(pelo Lema 2.21).

Agora vamos dividir a demonstração em dois casos:

CASO 1: $x_{n}$ é ímpar. 
Seja $q=\frac{x_{n}-1}{2}$. Então, por (2), temos que

$$
2 x_{n-1} \leq x_{n}=2 q+1<2(q+1)
$$

Logo, $x_{n-1}<q+1$ e, portanto, $x_{n-1} \leq q$ e de (1) teremos então

$$
-q \leq-x_{n-1}<\cdots<-x_{0}=-1<1=x_{0}<\cdots<x_{n-1} \leq q
$$

e como $-q, \ldots,-1,0,1, \ldots, q$ é um sistema completo de resíduos módulo $x_{n}$, as desigualdades acima mostram que

$$
-x_{n-1},-x_{n-2}, \ldots,-x_{0}, x_{0}, \ldots, x_{n-1}
$$

são incôngruos módulo $x_{n}$.

Além disso, como $\forall i \in \mathbb{N}, x_{i} \neq 0$, temos que

$$
-x_{n-1},-x_{n-2}, \ldots,-x_{0}, x_{0}, \ldots, x_{n-1}, x_{n}
$$

são incôngruos módulo $x_{n}$.

Desse fato, juntamente com (3), concluímos que

$$
x_{0}, x_{1}, \ldots, x_{n}, \ldots, x_{2 n}
$$

são incôngruos módulo $x_{n}$.

Portanto, se $x_{i} \equiv x_{j}\left(\bmod x_{n}\right), \operatorname{com} i \leq j \leq 2 n$, então $i=j$. 
CASO 2: $x_{n}$ é par.

Seja $q=\frac{x_{n}}{2}$. Então, por (2) temos que $x_{n-1} \leq \frac{x_{n}}{2}=q$ e de (1) teremos portanto que

$$
-q \leq-x_{n-1}<\cdots<-x_{0}=-1<1=x_{0}<\cdots<x_{n-1}=q .
$$

Se ocorrer $-q<-x_{n-1}$, então $-q+1 \leq-x_{n-1}$ e como $-q+1,-q+2, \ldots,-1,0,1, \ldots, q$ é um sistema completo de resíduos módulo $x_{n}$, como no caso anterior, teremos que $x_{0}, x_{1}, \ldots, x_{n}, \ldots, x_{2 n}$ são incôngruos módulo $x_{n}$. Mas se ocorrer $-q=-x_{n-1}$, então $x_{n-1}=q=\frac{x_{n}}{2}, \operatorname{logo}, 2 x_{n-1}=x_{n}$.

Por outro lado, do Lema 2.7, temos que $x_{n}=a x_{n-1}+d y_{n-1}$, logo

$$
2 x_{n-1}=x_{n}=a x_{n-1}+d y_{n-1} \geq a x_{n-1},
$$

mas como $a>1$, concluímos que $a=2$ e portanto $y_{n-1}=0$, ou seja, $n=1$. Mas, para $n=1$, a hipótese torna-se $x_{i} \equiv x_{j}\left(\bmod x_{1}\right)$, com $0 \leq i \leq j \leq 2$, e como $x_{0}=1$, $x_{1}=a=2, x_{2}=2 a^{2}-1=7$, por verificação direta, devemos ter $i=0$ e $j=2$.

Portanto, no caso $-x_{n-1}=-q$, devemos ter $a=2, n=1, i=0$ e $j=2$.

Lema 2.24 Se $x_{j} \equiv x_{i}\left(\bmod x_{n}\right)$, com $n>0,0<i \leq n, 0 \leq j<4 n$, então $j=i$ ou $j=4 n-i$.

Demonstração: CASO 1: $0<i \leq n \leq j \leq 2 n$.

Pelo Lema 2.23, se $i \neq j$, então $(n=1 \wedge a=2 \wedge i=0 \wedge j=2)$, contra $i>0$. Portanto, $i=j$. 
CASO 2: $0 \leq j<i \leq n<2 n$.

Pelo Lema 2.23, se $i \neq j$, então $(n=1 \wedge a=2 \wedge i=2 \wedge j=0)$, ou seja, $i=2>1=n$, contra $i \leq n$. Portanto, $i=j$.

CASO 3: $0<i \leq n<2 n<j<4 n$.

Seja $k=4 n-j$. Pelo Lema 2.22 e a hipótese, temos $x_{k}=x_{4 n-j} \equiv x_{j} \equiv x_{i}\left(\bmod x_{n}\right)$, e como $0<k<2 n$, pelo Lema 2.23 , se $i \neq k$, então devemos ter $a=2$ e $n=1$ e $[(i=0$ e $k=2)$ ou $(i=2, k=0)$ ], mas isso é impossível, pois $i, k>0$. Portanto, $i=k=4 n-j$, ou seja, $j=4 n-i$.

Lema 2.25 Se $0<i \leq n$ e $x_{j} \equiv x_{i}\left(\bmod x_{n}\right)$, então $j \equiv \pm i(\bmod 4 n)$.

Demonstração: Antes, mostremos por indução sobre $q$ que para dados $n>0$ e $r \geq 0$, vale $\forall q \geq 1, x_{4 n q+r} \equiv x_{r}\left(\bmod x_{n}\right)$.

De fato, para $q=1$, pelo Lema 2.22 , temos $x_{4 n+r} \equiv x_{r}\left(\bmod x_{n}\right)$. Agora, pelo Lema 2.22 , novamente temos que

$$
x_{4 n(q+1)+r}=x_{4 n+(4 n q+r)} \equiv x_{4 n q+r}\left(\bmod x_{n}\right)
$$

e por hipótese de indução, $x_{4 n q+r} \equiv x_{r}\left(\bmod x_{n}\right), \operatorname{logo}, x_{4 n(q+1)+r} \equiv x_{r}\left(\bmod x_{n}\right)$. Portanto, $\forall q \geq 1, x_{4 n q+r} \equiv x_{r}\left(\bmod x_{n}\right)$.

Agora, vamos ao resultado principal: façamos $j=4 n q+r$, com $0 \leq r<4 n$. Pelo resultado obtido acima e da hipótese, temos $x_{i} \equiv x_{j}=x_{4 n q+r} \equiv x_{r}\left(\bmod x_{n}\right)$. E como 
$0 \leq r<4 n$ e $0<i \leq n$, segue pelo Lema 2.24 que $i=r$ ou $i=4 n-r$ e portanto $j \equiv r \equiv \pm i(\bmod 4 n)$.

Lema 2.26 Se $b>a>1$, então $\forall n \geq 1$,

$$
x_{n}(b)>x_{n}(a) \quad \text { e } \quad y_{n}(b) \geq y_{n}(a)
$$

Demonstração: Por indução sobre $n$.

Base: $x_{1}(b)=b>a=x_{1}(a) \quad$ e $\quad y_{1}(b)=1=y_{1}(a)$.

Agora, pelo Lema 2.7, temos que

$$
\begin{aligned}
& x_{n+1}(b)=b x_{n}(b)+\left(b^{2}-1\right) y_{n}(b) \\
& x_{n+1}(a)=a x_{n}(a)+\left(a^{2}-1\right) y_{n}(a)
\end{aligned}
$$

Procedendo indutivamente, temos que

$$
x_{n+1}(b)=b x_{n}(b)+\left(b^{2}-1\right) y_{n}(b)>a x_{n}(a)+\left(a^{2}-1\right) y_{n}(a)=x_{n+1}(a) .
$$

Portanto, $x_{n}(b)>x_{n}(a)$. Agora, como $x_{n}^{2}(a)=1+d y_{n}^{2}(a)$ e $x_{n}^{2}(b)=1+d y_{n}(b)$ e $x_{n}(b)>x_{n}(a)$, segue que $y_{n}(b) \geq y_{n}(a)$. 


\section{Capítulo 3}

\section{A função exponencial é diofantina}

Teorema 3.1 Considere o sistema de equaçôes diofantinas:

$$
\begin{aligned}
& \text { (I) } x^{2}-\left(a^{2}-1\right) y^{2}=1 \\
& \text { (II) } u^{2}-\left(a^{2}-1\right) v^{2}=1 \\
& \text { (III) } s^{2}-\left(b^{2}-1\right) t^{2}=1 \\
& \text { (IV) } v=r y^{2} \\
& \text { (V) } b=1+4 p y=a+q u \\
& \text { (VI) } s=x+c u \\
& \text { (VII) } t=k+4(d-1) y \\
& \text { (VIII) } y=k+e-1
\end{aligned}
$$


Então, dados $x, k, a$, com $a>1$, existem $b, c, d, e, p, q, r, s, t, u, v, y$ que satisfazem o sistema (I) - (VIII) se e somente se $x=x_{k}(a)$.

\section{Demonstração: $(\Rightarrow)$}

Por (V), temos $b>a>1$, e de (I), (II), (III), pelo Lema 2.5 , existem $i, j, n$ tais que

$$
\begin{aligned}
& x=x_{i}(a), \quad y=y_{i}(a), \\
& u=x_{n}(a), \quad v=y_{n}(a), \\
& s=x_{j}(b), \quad t=y_{j}(b) .
\end{aligned}
$$

E como $y=y_{i}(a), v=y_{n}(a), t=y_{j}(a) \geq 1$, então $i, j, n>0$. Por (IV), $y \leq v$ e como $y_{k}(a)$ é uma função crescente, temos que

$$
i \leq n
$$

De $(\mathrm{V})$, temos que $b=a+q u \equiv a(\bmod u)$, ou seja, $b \equiv a\left(\bmod x_{n}(a)\right)$. Segue então do Lema 2.16 , que $x_{j}(b) \equiv x_{j}(a)\left(\bmod x_{n}(a)\right)$ e como de (VI), $s=x+c u \equiv x(\bmod u)$, ou seja, $x_{j}(b) \equiv x_{i}(a)\left(\bmod x_{n}(a)\right)$, temos então que

$$
x_{i}(a) \equiv x_{j}(a)\left(\bmod x_{n}(a)\right)
$$

Segue de (3.1) e (3.2) e do Lema 2.25 que

$$
j \equiv \pm i(\bmod 4 n)
$$

Agora, de (IV) temos que $y^{2} \mid v$, ou seja, $\left(y_{i}(a)\right)^{2} \mid y_{n}(a)$ que pelo Lema 2.13, resulta que $y_{i}(a) \mid n$, e junto com (3.3), obtemos

$$
j \equiv \pm i\left(\bmod 4 y_{i}(a)\right.
$$


Por $(\mathrm{V}), b=1+4 p y \equiv 1(\bmod 4 y)$, isto é, $b-1 \equiv 0\left(\bmod 4 y_{i}(a)\right)$, e como pelo Lema $2.15, y_{j}(b) \equiv j(\bmod b-1)$, segue que

$$
y_{j}(b) \equiv j\left(\bmod 4 y_{i}(a)\right)
$$

Agora, por (VII) temos $t=k+4(d-1) y \equiv k(\bmod 4 y)$, isto é,

$$
y_{j}(b) \equiv k\left(\bmod 4 y_{i}(a)\right)
$$

Então de (3.4), (3.5) e (3.6), temos que

$$
k \equiv \pm i\left(\bmod 4 y_{i}(a)\right)
$$

Por (VIII), $k \leq y$, isto é,

$$
k \leq y_{i}(a)<4 y_{i}(a)
$$

Além disso, pelo Lema 2.19, $i \leq y_{i}(a)<2 y_{i}(a)$. Então, $-2 y_{i}(a)<-i$, logo $-2 y_{i}(a)+1$ $\leq-i$ e como $-2 y_{i}(a)+1, \ldots,-1,0,1, \ldots, 2 y_{i}(a)$ é um sistema completo de resíduos módulo $4 y_{i}(a)$, segue juntamente de (3.7) e (3.8) que $k=1$. Portanto, $x=x_{i}(a)=x_{k}(a)$.

$$
(\Leftarrow)
$$

Seja então $x=x_{k}(a)$, e façamos $y=y_{k}(a)$. Como $k>0$, então $x, y>0$ e (I) está satisfeita.

Seja agora $m=2 k y_{k}(a)>0$ e façamos $u=x_{m}(a)>0$ e $v=y_{m}(a)>0$. Então (II) está satisfeita.

Como $k y_{k}(a) \mid 2 k y_{k}(a)$, então pelo Lema 2.10, temos que $y_{k y_{k}(a)}(a) \mid y_{2 k y_{k}(a)}(a)$, e como pelo Lema $2.12, y_{k}^{2}(a) \mid y_{k y_{k}(a)}(a)$, resulta então que $y_{k}^{2}(a) \mid y_{2 k y_{k}(a)}(a)=y_{m}(a)$, isto é, $y^{2} \mid v$. Portanto, existe $r>0$ tal que $v=r y^{2}$, e (IV) está satisfeita. 
Além disso, como $m=2 k y_{k}(a)$ é par, pelo Lema 2.17, $y_{m}(a)=v$ é par, e como (II) já está satisfeita, isto é, $u^{2}-\left(a^{2}-1\right) v^{2}=1$, temos que $u^{2}$ é ímpar, logo $u$ é ímpar, e portanto $\operatorname{mdc}(u, v)=1$.

Afirmamos agora que $\operatorname{mdc}(u, 4 y)=1$.

De fato, seja $p$ um primo tal que $p \mid u$ e $p \mid 4 y$ como $u$ é ímpar, então $p \neq 2$, logo $p \nmid 4$. Portanto, $p \mid y$, e como $y \mid v$, teríamos $p \mid v$, de forma que $p \mid u$ e $p \mid v$, mas como já vimos, $\operatorname{mdc}(u, v)=1$. Portanto, $\operatorname{mdc}(u, 4 y)=1$.

Pelo teorema chinês do resto, existe então $b_{0}$ tal que

$$
\left\{\begin{array}{l}
b_{0} \equiv 1(\bmod 4 y) \\
b_{0} \equiv a(\bmod u)
\end{array}\right.
$$

Seja $j_{0}=\min \left\{j \geq 0: b_{0}+4 j u y>a\right\}$ e façamos $b=b_{0}+4 j_{0} u y$. É fácil ver então que $b>a>1 \mathrm{e}$

$$
\left\{\begin{array}{l}
b \equiv 1(\bmod 4 y) \\
b \equiv a(\bmod u)
\end{array}\right.
$$

Portanto, existem $b, p, q>0$ tais que $b=1+4 p y=a+q u$ e (V) está satisfeita.

Como já vimos, $b>a>1$, então fazemos $s=x_{k}(b)>0$ e $t=y_{k}(b)>0$ e (III) está satisfeita.

Usando novamente que $b>a$, pelo Lema 2.26 temos que $s=x_{k}(b)>x_{k}(a)=x$. Além disso, como de $(\mathrm{V}) b=a+q u \equiv a(\bmod u)$, pelo Lema 2.16 , temos que

$$
x_{k}(b) \equiv x_{k}(a)(\bmod u)
$$

ou seja, $s \equiv x(\bmod u)$. Como $s>x$, existe $c>0$ tal que $s=x+c u$, e (VI) está satisfeita. 
Agora, pelo Lema $2.15, y_{k}(b) \equiv k(\bmod b-1)$, ou seja, $t \equiv k(\bmod b-1)$, e por $(\mathrm{V})$, $b=1+4 p y, \operatorname{logo} 4 y \mid b-1$, e desses dois fatos, temos que $t \equiv k(\bmod 4 y)$. Além disso, pelo Lema $2.19, y_{k}(b) \geq k$, ou seja, $t \geq k$, e então existe $d>0$ tal que $t=k+4(d-1) y$ e (VII) está satisfeita.

Finalmente, pelo Lema 2.19, $y_{k}(a) \geq k$, isto é, $y \geq k$, portanto existe $e>0$ tal que $e=y-k+1, \mathrm{e}(\mathrm{VIII})$ está satisfeita.

Corolário 3.2 A função $g(z, k)=x_{k}(z+1)$ é diofantina.

Demonstração: Seja $S$ o sistema consistindo das equações (I) - (VIII) do Teorema 3.1 mais a equação $a=z+1$. Então, pelo Teorema 3.1, $S$ admite solução se e somente se $x=x_{k}(a)=x_{k}(z+1)=g(z, k)$. Portanto, a equação diofantina que define a função $g$ é obtida de maneira usual pela soma dos quadrados de nove polinômios obtidos a partir do sistema $S$.

Lema 3.3 Seja $a>1, n, k>0$. Se $a>n^{k}$, então $2 a n-n^{2}-1>n^{k}$.

Demonstração: Seja $P(n) \equiv\left(1 \leq n \leq a \Rightarrow 2 a n-n^{2}-1 \geq a\right)$. Mostremos que $\forall n>0$, $P(n)$ por indução sobre $n$ :

Base: $n=1$.

Então $2 a n-n^{2}-1=2 a-2 \geq a$, pois como $2 \leq a$, então $a+2 \leq a+a=2 a$, logo, $a \leq 2 a-2$. Suponha agora que $1 \leq n+1 \leq a$. Isso implica que $1 \leq n \leq a$, já que $n>0$. 
Logo, por hipótese de indução, $2 a n-n^{2}-1 \geq a$. Então,

$$
\begin{aligned}
& 2 a(n+1)-(n+1)^{2}-1=2 a n+2 a-n^{2}-2 n-1-1 \\
& =\left(2 a n-n^{2}-1\right)+(2 a-2 n-1)>a+0=a,
\end{aligned}
$$

pois como $1 \leq n \leq a$, então $1 \leq n-a<2 n-2 a$, logo $2 n-2 a-1>0$.

Portanto, $\forall n \geq 1, P(n)$.

Agora é imediato que se $a>n^{k}$, então $a>n$ e pelo resultado acima, temos que $n^{k}<a \leq 2 a n-n^{2}-1$.

Lema 3.4 Considere as equações

$$
\begin{aligned}
& \text { (IX) }(x-y(a-n)-m)^{2}=(f-1)^{2}\left(2 a n-n^{2}-1\right)^{2} \\
& \text { (X) } m+g=2 a n-n^{2}-1 \\
& \text { (XI) } w=n+h=k+l \\
& \text { (XII) } a^{2}-\left(w^{2}-1\right)(w-1)^{2} z^{2}=1
\end{aligned}
$$

e seja $S$ o sistema consistindo das equações (I) - (VIII) do Teorema 3.1 e de (IX) - (XII). Então existem $a, b, c, d, e, f, g, h, l, p, q, r, s, t, u, v, x, y, z, w$ que satisfazem o sistema $S$ se e somente se $m=n^{k}$. 


\section{Demonstração: $(\Rightarrow)$}

Por (XI), $w>1$, logo $\left(w^{2}-1\right)(w-1)^{2} z^{2}>0$, então por (XII), $a>1$, e segue do Teorema 3.1 que $x=x_{k}(a)$ e portanto, $y=y_{k}(a)$.

Do Lema $2.18, x_{k}(a)-y_{k}(a)(a-n) \equiv n^{k}\left(\bmod 2 a n-n^{2}-1\right)$ e de (IX),

$$
x_{k}(a)-y_{k}(a)(a-n)= \pm(f-1)\left(2 a n-n^{2}-1\right)+m,
$$

portanto $m \equiv n^{k}\left(\bmod 2 a n-n^{2}-1\right)$.

Afirmamos agora que $n^{k}<2 a n-n^{2}-1$.

De fato, por (XII), como $w>1$, temos que $\operatorname{Pell}_{w}(a,(w-1) z)$ e então, pelo Lema 2.5, existe $j$ tal que $a=x_{j}(w)$ e $(w-1) z=y_{j}(w)$. Além disso, pelo Lema 2.15, $y_{j}(w)=$ $(w-1) z \equiv j(\bmod w-1) . \operatorname{Logo}, j \equiv 0(\bmod w-1)$, e portanto $w-1 \leq j$. Agora, pelo Lema $2.20, x_{j}(w)=a \geq w^{j}, \operatorname{logo}, a \geq w^{j} \geq w^{w-1}$, e como por (XI) temos que $k, n<w$, resulta que

$$
a \geq w^{j} \geq w^{w-1} \geq w^{k}>n^{k}
$$

Portanto, $a>n^{k}$ e, então pelo Lema 3.4, temos que $n^{k}<2 a n-n^{2}-1$. Como de (X) $m<2 a n-n^{2}-1$, e como já vimos que $m \equiv n^{k}\left(\bmod 2 a n-n^{2}-1\right)$, temos então que $m=n^{k}$.

$(\Leftarrow)$ Seja $m=n^{k}$.

Tomemos $w$ tal que $w>n, k$ e seja $a=x_{w-1}(w)$. Como $n, k \geq 1$, então $w-1>0$ e portanto $a=x_{w-1}(w)>1$. Pelo Lema $2.15, y_{w-1}(w) \equiv w-1(\bmod w-1), \operatorname{logo}$ 
$y_{w-1}(w) \equiv 0(\bmod w-1)$, e como $w-1>0$, temos que $y_{w-1}(w)>0$, então existe $z>0$ tal que $y_{w-1}(w)=z(w-1)$. Portanto, temos $\operatorname{Pell}_{w}(a, z(w-1))$ e (XII) está satisfeita.

Como $w>n, k$, existem $h, l>0$ tais que $w=n+h$ e $w=k+l$, e (XI) está satisfeita.

Agora pelo Lema 2.20, $a=x_{w-1}(w) \geq w^{w-1}$, e como $w>n, k$, temos que

$$
a=x_{w-1}(w) \geq w^{w-1} \geq w^{k}>n^{k} .
$$

Então, $a>n^{k}$ e pelo Lema $3.3, m=n^{k}<2 a n-n^{2}-1$, e portanto existe $g>0$ tal que $m+g=2 a n-n^{2}-1, \mathrm{e}(\mathrm{X})$ está satisfeita.

Façamos agora $x=x_{k}(a)$ e $y=y_{k}(a)$. Pelo Lema 2.18,

$$
x_{k}(a)-y_{k}(a)(a-n) \equiv n^{k}=m\left(\bmod 2 a n-n^{2}-1\right)
$$

Portanto existe $f>0$ tal que

$$
x_{k}(a)-y_{k}(a)(a-n)-m= \pm(f-1)\left(2 a n-n^{2}-1\right),
$$

e (IX) está satisfeita.

Finalmente, como $a>1$, e $x=x_{k}(a)$, pelo Teorema 3.1, as equações (I) - (VIII) estão satisfeitas.

Teorema 3.5 A função exponencial $h(n, k)=n^{k}$ é diofantina.

Demonstração: Pelo Lema 3.4, obtemos o polinômio associado à função $h(n, k)=n^{k}$ a partir da soma dos quadrados dos polinômios presentes nas equações (I) - (XII). 


\section{Capítulo 4}

\section{As funções $\left(\begin{array}{l}n \\ k\end{array}\right), n ! \mathbf{e} \prod_{k=1}^{n}(a+b k)$ são} diofantinas

Lema 4.1 Se $0<k \leq n$ e $u>2^{n}$, então

$$
q\left((u+1)^{n}, u^{k}\right)=\sum_{i=k}^{n}\left(\begin{array}{l}
n \\
i
\end{array}\right) u^{i-k},
$$

onde $q(x, y)$ é o quociente de $x$ por $y$.

Demonstração: Pelo algoritmo da divisão, basta mostrar que existe $R$ tal que

$$
(u+1)^{n}=u^{k} \sum_{i=k}^{n}\left(\begin{array}{c}
n \\
i
\end{array}\right) u^{i-k}+R, \text { com } 0 \leq R<u^{k} .
$$

Afirmamos que $R=\sum_{i=0}^{k-1}\left(\begin{array}{c}n \\ i\end{array}\right) u^{i} \geq 0$. De fato, pelo teorema do binômio, temos que

$$
\begin{aligned}
(u+1)^{n} & =\sum_{i=0}^{n}\left(\begin{array}{c}
n \\
i
\end{array}\right) u^{i}=\sum_{i=k}^{n}\left(\begin{array}{c}
n \\
i
\end{array}\right) u^{i}+\sum_{i=0}^{k-1}\left(\begin{array}{c}
n \\
i
\end{array}\right) u^{i} \\
& =u^{k} \sum_{i=k}^{n}\left(\begin{array}{c}
n \\
i
\end{array}\right) u^{i-k}+\sum_{i=0}^{k-1}\left(\begin{array}{c}
n \\
i
\end{array}\right) u^{i}
\end{aligned}
$$


Basta mostrar agora que $\sum_{i=0}^{k-1}\left(\begin{array}{l}n \\ i\end{array}\right) u^{i}<u^{k}$. De fato, como por hipótese $0<k \leq n$ e $u>2^{n}$, temos

$$
\begin{aligned}
\sum_{i=0}^{k-1}\left(\begin{array}{c}
n \\
i
\end{array}\right) u^{i} & \leq u^{k-1} \sum_{i=0}^{k-1}\left(\begin{array}{c}
n \\
i
\end{array}\right)<u^{k-1} \sum_{i=0}^{n}\left(\begin{array}{l}
n \\
i
\end{array}\right) \\
& =u^{k-1} 2^{n}<u^{k-1} u=u^{k} .
\end{aligned}
$$

Lema 4.2 Se $0<k \leq n$ e $u>2^{n}$, então

$$
q\left((u+1)^{n}, u^{k}\right) \equiv\left(\begin{array}{l}
n \\
k
\end{array}\right) \quad(\bmod u)
$$

Demonstração: Basta usar o Lema 4.1 e notar que

$$
q\left((u+1)^{n}, u^{k}\right)=\sum_{i=k}^{n}\left(\begin{array}{l}
n \\
i
\end{array}\right) u^{i-k}=\left(\begin{array}{l}
n \\
k
\end{array}\right)+u \sum_{i=k+1}^{n}\left(\begin{array}{l}
n \\
i
\end{array}\right) u^{i-k-1} .
$$

Teorema 4.3 A função $f(n, k)=\left(\begin{array}{l}n \\ k\end{array}\right)$ é diofantina.

Demonstração: Dados $n, k, \operatorname{com} 0<k \leq n$, tomemos $u$ tal que $u>2^{n}$. Pelo Lema 4.2, temos que

$$
q\left((u+1)^{n}, u^{k}\right) \equiv\left(\begin{array}{l}
n \\
k
\end{array}\right)(\bmod u) .
$$

Além disso, $\left(\begin{array}{l}n \\ k\end{array}\right) \leq \sum_{i=0}^{n}\left(\begin{array}{l}n \\ i\end{array}\right)=2^{n}<u$. Isso mostra que $\left(\begin{array}{l}n \\ k\end{array}\right)$ é o único número inteiro positivo congruente a $q\left((u+1)^{n}, u^{k}\right)$ módulo $u$. Portanto,

$$
\begin{aligned}
z=\left(\begin{array}{l}
n \\
k
\end{array}\right) \Leftrightarrow \exists u, v, w, r, s[v & =2^{n} \wedge u>v \wedge r=(u+1)^{n} \wedge s=u^{k} \\
\wedge w & =q(r, s) \wedge z<u \wedge z \equiv w(\bmod u)] .
\end{aligned}
$$


Isso mostra que a função $f(n, k)=\left(\begin{array}{l}n \\ k\end{array}\right)$ é diofantina, pois cada fórmula da expressão à direita, ligadas pelo conectivo $\wedge$, são diofantinas. De fato: $v=2^{n}, r=(u+1)^{n}$ e $s=u^{k}$ são diofantinas pelo Teorema 3.5. Além disso,

$$
\begin{aligned}
u>v & \Leftrightarrow \exists x(u=v+x), \\
w=q(r, s) & \Leftrightarrow \exists x, y(r=s x+y \wedge 0 \leq y<s) .
\end{aligned}
$$

Finalmente,

$$
z \equiv w(\bmod u) \wedge z<u \Leftrightarrow \exists x, y(w=z+(x-1) u \wedge u=z+y)
$$

Lema 4.4 Se $1 \leq x<r$, então $\left(\begin{array}{l}r \\ x\end{array}\right) x ! \leq r^{x}$

Demonstração: $\left(\begin{array}{l}r \\ x\end{array}\right) x !=\frac{r !}{(r-x) !}=\prod_{i=0}^{x-1}(r-i) \leq \prod_{i=0}^{x-1} r=r^{x}$.

Lema 4.5 Se $1 \leq x<r$, então $(r-x)^{x}<\frac{r !}{(r-x) !}$.

Demonstração: $(r-x)^{x}=\prod_{i=0}^{x-1}(r-x)<\prod_{i=0}^{x-1}(r-i)=\frac{r !}{(r-x) !}$.

Lema 4.6 Se $x \geq 1$ e $2 x<r$, então $\left(r^{x}\right)^{2}<(r-x)^{x}(r+2 x)^{x}$.

Demonstração: Por hipótese, $r-2 x>0$, e como $x \geq 1$, temos que

$$
x(r-2 x)=x r-2 x^{2}>0,
$$


logo, $r^{2}<r^{2}+x r-2 x^{2}$, e como $x \geq 1$, temos

$$
\begin{aligned}
\left(r^{x}\right)^{2} & =\left(r^{2}\right)^{x}<\left(r^{2}+x r-2 x^{2}\right)^{x} \\
& =[(r-x)(r+2 x)]^{x}=(r-x)^{k}(r+2 x)^{x}
\end{aligned}
$$

Lema 4.7 Se $x \geq 1$ e $2 x<r$, então $(r+2 x)^{x}<r^{x}+r^{x-1} 2 x 2^{x}$.

Demonstração: Pelo teorema do binômio:

$$
\begin{aligned}
(r+2 x)^{x} & =\sum_{i=0}^{x}\left(\begin{array}{c}
x \\
i
\end{array}\right) r^{x-i}(2 x)^{i}=r^{x}+\sum_{i=1}^{x}\left(\begin{array}{l}
x \\
i
\end{array}\right) r^{x-i}(2 x)^{i} \\
& =r^{x}+\sum_{i=1}^{x}\left(\begin{array}{c}
x \\
i
\end{array}\right) r^{x-i}(2 x)^{i-1} 2 x \\
\stackrel{\text { Hip. }}{\leq} & r^{x}+\sum_{i=1}^{x}\left(\begin{array}{c}
x \\
i
\end{array}\right) r^{x-i} r^{i-1} 2 x=r^{x}+\sum_{i=1}^{x}\left(\begin{array}{c}
x \\
i
\end{array}\right) r^{x-1} 2 x \\
& =r^{x}+r^{x-1} 2 x \sum_{i=1}^{x}\left(\begin{array}{c}
x \\
i
\end{array}\right)<r^{x}+r^{x-1} 2 x \sum_{i=0}^{x}\left(\begin{array}{c}
x \\
i
\end{array}\right) \\
& =r^{x}+r^{x-1} 2 x 2^{x} .
\end{aligned}
$$

Lema 4.8 Se $x \geq 1$ e $(2 x)^{x+1}<r$, então $r^{x}<\left(\begin{array}{l}r \\ x\end{array}\right)(x !+1)$.

Demonstração: Notemos que como $(2 x)^{x+1}<r$, as condições dos Lemas $4.4,4.5$ e 4.6 estão satisfeitas.

Pelo Lema 4.6, $\left(r^{x}\right)^{2}<(r-x)^{x}(r+2 x)^{x}$, e portanto

$$
\left(\begin{array}{l}
r \\
x
\end{array}\right) x !\left(r^{x}\right)^{2}<\left(\begin{array}{l}
r \\
x
\end{array}\right) x !(r-x)^{x}(r+2 x)^{x}
$$


Por outro lado, pelo Lema $4.5,(r-x)^{x}<\frac{r !}{(r-x) !}$. Então,

$$
\left(r^{x}\right)^{2}(r-x)^{x}<\frac{r !}{(r-x) ! x !} x !\left(r^{x}\right)^{2}=\left(\begin{array}{l}
r \\
x
\end{array}\right) x !\left(r^{x}\right)^{2}
$$

De (4.1) e (4.2), obtemos

$$
\left(r^{x}\right)^{2}(r-x)^{2}<\left(\begin{array}{l}
r \\
x
\end{array}\right) x !(r-x)^{x}(r+2 x)^{x}
$$

e portanto $\left(r^{x}\right)^{2}<\left(\begin{array}{l}r \\ x\end{array}\right) x !(r+2 x)^{x}$ e como pelo Lema 4.7, $(r+2 x)^{x}<r^{x}+r^{x-1} 2 x 2^{x}$, temos que

$$
\left(r^{x}\right)^{2}<\left(\begin{array}{l}
r \\
x
\end{array}\right) x !\left(r^{x}+r^{x-1} 2 x 2^{x}\right)
$$

ou seja,

$$
\left(r^{x-1}\right)\left(r^{x+1}\right)=\left(r^{x}\right)^{2}<\left(\begin{array}{l}
r \\
x
\end{array}\right) x ! r^{x-1}\left(r+2 x 2^{x}\right)
$$

e portanto, teremos que

$$
r^{x+1}<\left(\begin{array}{l}
r \\
x
\end{array}\right)\left(r x !+x ! 2 x 2^{x}\right)
$$

e como $x ! \leq x^{x}$, resulta que

$$
r^{x+1}<\left(\begin{array}{l}
r \\
x
\end{array}\right)\left(r x !+x^{x} 2 x 2^{x}\right)=\left(\begin{array}{l}
r \\
x
\end{array}\right)\left(r x !+(2 x)^{x+1}\right)
$$

e como por hipótese $(2 x)^{x+1}<r$, temos que

$$
r^{x+1}<\left(\begin{array}{l}
r \\
x
\end{array}\right)(r x !+r)=\left(\begin{array}{l}
r \\
x
\end{array}\right) r(x !+1),
$$

de onde obtemos por fim que

$$
r<\left(\begin{array}{l}
r \\
x
\end{array}\right)(x !+1)
$$

Lema 4.9 Se $x \geq 1$ e $(2 x)^{x+1}<r$, então $q\left(r^{x},\left(\begin{array}{l}r \\ x\end{array}\right)\right)=x$ !. 
Demonstração: Pelo algoritmo da divisão, devemos mostrar que existe um único $R$ tal que

$$
r^{x}=\left(\begin{array}{l}
r \\
x
\end{array}\right) x !+R, \text { com } 0 \leq R<\left(\begin{array}{l}
r \\
x
\end{array}\right) .
$$

Afirmamos que $R=r^{x}-\left(\begin{array}{l}r \\ x\end{array}\right) x$ !. Devemos então mostrar que

$$
0 \leq r^{x}-\left(\begin{array}{l}
r \\
x
\end{array}\right) x !<\left(\begin{array}{l}
r \\
x
\end{array}\right)
$$

ou ainda

$$
\left(\begin{array}{l}
r \\
x
\end{array}\right) x ! \leq r^{x}<\left(\begin{array}{l}
r \\
x
\end{array}\right)(x !+1),
$$

e essas desigualdades são dadas pelos Lemas 4.4 e 4.8 .

Teorema $4.10 \mathrm{~g}(n)=n$ ! é uma função diofantina.

Demonstração: Vimos no Lema 4.9 que se $r>(2 n)^{n+1}$, então $q\left(r^{n},\left(\begin{array}{l}r \\ n\end{array}\right)\right)=n !$, portanto,

$$
\begin{gathered}
m=n ! \Leftrightarrow \exists s, t, w, r, u, v\left[s=2 n \wedge t=n+1 \wedge w=s^{t} \wedge r=w+1\right. \\
\left.\wedge u=r^{n} \wedge v=\left(\begin{array}{l}
r \\
n
\end{array}\right) \wedge q(u, v)=m\right] .
\end{gathered}
$$

Isso mostra que $g(n)=n$ ! é uma função diofantina, pois como já vimos, $s=2 n, t=n+1$, $w=s^{t}, r=w+1, u=r^{n}, q(u, v)=m$ são diofantinas e $v=\left(\begin{array}{l}r \\ n\end{array}\right)$ é diofantina pelo Teorema 4.3 .

Lema 4.11 Se $b q \equiv a(\bmod m)$, então

$$
\prod_{k=1}^{y}(a+b k) \equiv b^{y} y !\left(\begin{array}{c}
q+y \\
y
\end{array}\right) \quad(\bmod m)
$$




\section{Demonstração:}

$$
\begin{aligned}
b^{y} y !\left(\begin{array}{c}
q+y \\
y
\end{array}\right) & =b^{y} \frac{(q+y) !}{q !}=b^{y} \prod_{i=0}^{y-1}(q+(y-i)) \\
& =\prod_{i=0}^{y-1}(b q+b(y-i)) \equiv \prod_{i=0}^{y-1}(a+b(y-i)) \\
& =\prod_{k=1}^{y}(a+b k)(\bmod m)
\end{aligned}
$$

Teorema $4.12 h(a, b, y)=\prod_{k=1}^{y}(a+b k)$ é uma função diofantina.

Demonstração: Para dados $a, b, y$, seja $m=b(a+b y)^{y}+1$. É claro que $\operatorname{mdc}(m, b)=1$ e portanto existe $q$ que satisfaz a congruência $b q \equiv a(\bmod m)$. Pelo Lema 4.11, segue então que

$$
\prod_{k=1}^{y}(a+b k) \equiv b^{y} y !\left(\begin{array}{c}
q+y \\
y
\end{array}\right)(\bmod m)
$$

Além disso, claramente

$$
\prod_{k=1}^{y}(a+b k)<b(a+b y)^{y}+1=m .
$$

Portanto, $\prod_{k=1}^{y}(a+b k)$ é o único inteiro positivo congruente a $b^{y} y !\left(\begin{array}{c}q+y \\ y\end{array}\right)$ módulo $m$. Segue então que

$$
z=\prod_{k=1}^{y}(a+b k)
$$

$$
\begin{aligned}
& \exists m, p, q, r, s, t, u, v, w, x\left[r=a+b y \wedge s=r^{y} \wedge m=b s+1\right. \\
& \wedge b q=a+m t \wedge u=b^{y} \wedge v=y ! \wedge z<m \wedge w=q+y \\
& \left.\wedge x=\left(\begin{array}{l}
w \\
y
\end{array}\right) \wedge z+m p=u v x\right]
\end{aligned}
$$


e isso mostra que $h(a, b, y)=\prod_{k=1}^{y}(a+b k)$ é diofantina, pois $v=y$ ! é diofantina pelo Teorema 4.10 e as fórmulas restantes, como já comentamos, são diofantinas. 


\section{Capítulo 5}

\section{Quantificadores limitados são diofantinos}

Lema 5.1 Seja $P\left(y, k, x_{1}, \ldots, x_{n}, y_{1}, \ldots, y_{m}\right)$ um polinômio. Entâo

$$
\begin{gathered}
(\forall k)_{\leq y} \exists y_{1}, \ldots, y_{m}\left(P\left(y, k, x_{1}, \ldots, x_{n}, y_{1}, \ldots, y_{m}\right)=0\right) \\
\mathbb{W} \\
\exists u(\forall k)_{\leq u}\left(\exists y_{1}, \ldots, y_{m}\right)_{\leq u}\left(P\left(y, k, x_{1}, \ldots, x_{n}, y_{1}, \ldots, y_{m}\right)=0\right)
\end{gathered}
$$

Demonstração: (介) Imediato.

$(\Downarrow)$ A hipótese afirma que para cada $k=1, \ldots, y$ existem $y_{1}^{(k)}, \ldots, y_{m}^{(k)}$ tais que

$$
P\left(y, k, x_{1}, \ldots, x_{n}, y_{1}^{(k)}, \ldots, y_{m}^{(k)}\right)=0
$$

Seja $u=\max \left\{y_{j}^{(k)}: j=1, \ldots, m\right.$ e $\left.k=1, \ldots, y\right\}$. Portanto,

$$
\exists u(\forall k)_{\leq y}\left(\exists y_{1}, \ldots, y_{m}\right)_{\leq u}\left(P\left(y, k, x_{1}, \ldots, x_{n}, y_{1}, \ldots, y_{m}\right)=0\right)
$$


Lema 5.2 Dado um polinômio $P\left(y, k, x_{1}, \ldots, x_{n}, y_{1}, \ldots, y_{m}\right)$, seja $Q\left(y, u, x_{1}, \ldots, x_{n}\right)$ um polinômio tal que

(1) $Q\left(y, u, x_{1}, \ldots, x_{n}\right)>u$;

(2) $Q\left(y, u, x_{1}, \ldots, x_{n}\right)>y$;

(3) $k \leq y$ e $y_{1}, \ldots, y_{m} \leq u \Rightarrow\left|P\left(y, k, x_{1}, \ldots, x_{n}, y_{1}, \ldots, y_{m}\right)\right| \leq Q\left(y, u, x_{1}, \ldots, x_{n}\right)$.

\section{Então}

$$
\begin{gathered}
(\forall k)_{\leq y}\left(\exists y_{1}, \ldots, y_{m}\right)_{\leq u}\left(P\left(y, k, x_{1}, \ldots, x_{n}, y_{1}, \ldots, y_{m}\right)=0\right) \\
\mathbb{1} \\
\exists c, t, a_{1}, \ldots, a_{m}\left[1+c t=\prod_{k=1}^{y}(1+k t) \wedge t=Q\left(y, u, x_{1}, \ldots, x_{n}\right) !\right. \\
\wedge 1+c t\left|\prod_{j=1}^{u}\left(a_{1}-j\right) \wedge \ldots \wedge 1+c t\right| \prod_{j=1}^{u}\left(a_{m}-j\right) \\
\left.\wedge P\left(y, c, x_{1}, \ldots, x_{n}, a_{1}, \ldots, a_{m}\right) \equiv 0(\bmod 1+c t)\right]
\end{gathered}
$$

Demonstração: $(\Downarrow)$ Para cada $k=1,2, \ldots, y$ e $j=1,2, \ldots, m$, sejam $y_{j}^{(k)}$ tais que por hipótese $y_{j}^{(k)} \leq u$ e $P\left(y, k, x_{1}, \ldots, x_{n}, y_{1}^{(k)}, \ldots, y_{m}^{(k)}\right)=0$. Seja

$$
t=Q\left(y, u, x_{1}, \ldots, x_{n}\right) !
$$

Como $\prod_{k=1}^{y}(1+k t) \equiv 1(\bmod t)$, então existe $c>0$ tal que

$$
1+c t=\prod_{k=1}^{y}(1+k t)
$$


Afirmamos agora que para $1 \leq k<l \leq y, \operatorname{mdc}(1+k t, 1+l t)=1$. De fato, suponha que $p$ é um primo tal que $p \mid 1+k t$ e $p \mid 1+l t$. Então $p \mid l-k$ e como $0<l-k<y$, então $p<y$. Mas, pela hipótese (2), $Q\left(y, u, x_{1}, \ldots, x_{n}\right)>y, \operatorname{logo} Q\left(y, u, x_{1}, \ldots, x_{n}\right)>p$ e, portanto, $p \mid Q\left(y, u, x_{1}, \ldots, x_{n}\right)$ !, ou seja, $p \mid t$, e como $p \mid 1+k t$, isso implica que $p \mid 1$. Absurdo.

Portanto, para $1 \leq k<l \leq y, \operatorname{mdc}(1+k t, 1+l t)=1)$.

Podemos então aplicar o teorema chinês do resto nos $m$ sistemas

$$
\begin{gathered}
\left\{\begin{array}{cc}
x \equiv y_{1}^{(1)} & (\bmod 1+1 t) \\
x \equiv y_{1}^{(2)} & (\bmod 1+2 t) \\
\vdots & \\
x \equiv y_{1}^{(y)} & (\bmod 1+y t)
\end{array}\right. \\
\vdots \\
\left\{\begin{array}{cc}
x \equiv y_{m}^{(1)} & (\bmod 1+1 t) \\
x \equiv y_{m}^{(2)} & (\bmod 1+2 t) \\
\vdots & \\
x \equiv y_{m}^{(y)} & (\bmod 1+y t)
\end{array}\right.
\end{gathered}
$$

e obter uma solução $a_{i}$ para cada um dos sistemas, ou seja, para $i=1, \ldots, m$, teremos que

$$
a_{i} \equiv y_{i}^{(k)}(\bmod 1+k t), \quad k=1, \ldots, y
$$

Por outro lado, como $1+c t=\prod_{k=1}^{y}(1+k t)$, então, para $k=1, \ldots, y$, temos

$$
1+c t \equiv 0(\bmod 1+k t)
$$

donde $k+k c t \equiv 0(\bmod 1+k t)$. Também, para $k=1, \ldots, y$,

$$
1+k t \equiv 0(\bmod 1+k t)
$$

donde $c+k c t \equiv 0(\bmod 1+k t)$. 
Portanto, para $k=1, \ldots, y, c \equiv k(\bmod 1+k t)$.

As congruências $a_{i} \equiv y_{i}^{(k)}(\bmod 1+k t)$ e $c \equiv k(\bmod 1+k t)$ permitem-nos afirmar que para $k=1, \ldots, y$ :

$$
P\left(y, c, x_{1}, \ldots, x_{n}, a_{1}, \ldots, a_{m}\right) \equiv P\left(y, k, x_{1}, \ldots, x_{n}, y_{1}^{(k)}, \ldots, y_{m}^{(k)}\right)=0(\bmod 1+k t)
$$

e como $k \neq l \Rightarrow \operatorname{mdc}(1+k t, 1+l t)=1$, temos que

$$
P\left(y, c, x_{1}, \ldots, x_{n}, a_{1}, \ldots, a_{m}\right) \equiv 0 \quad\left(\bmod \prod_{k=1}^{y}(1+k t)\right)
$$

ou seja,

$$
P\left(y, c, x_{1}, \ldots, x_{n}, a_{1}, \ldots, a_{m}\right) \equiv 0(\bmod 1+c t)
$$

Finalmente, como para $i=1, \ldots, m$ vale

$$
a_{i} \equiv y_{i}^{(k)}(\bmod 1+k t), \quad k=1, \ldots, y
$$

então $1+k t \mid a_{i}-y_{i}^{(k)}, k=1, \ldots, y$, e como $1 \leq y_{i}^{(k)} \leq u$, isso implica que

$$
1+k t \mid \prod_{j=1}^{u}\left(a_{i}-j\right), \text { para } k=1, \ldots, y .
$$

Usando novamente o fato de que $k \neq l \Rightarrow \operatorname{mdc}(1+k t, 1+l t)=1$, teremos que

$$
\prod_{k=1}^{y}(1+k t) \mid \prod_{j=1}^{u}\left(a_{i}-j\right)
$$

ou seja,

$$
1+c t\left|\prod_{j=1}^{u}\left(a_{1}-j\right) \wedge \cdots \wedge 1+c t\right| \prod_{j=1}^{u}\left(a_{m}-j\right) .
$$

( $\uparrow$ Para cada $k=1, \ldots, y$, e para cada $i=1, \ldots, m$, seja $p_{k}$ um primo tal que $p_{k} \mid 1+k t$ e seja $y_{i}^{(k)}=r\left(a_{i}, p_{k}\right)$ (resto da divisão de $a_{i}$ por $p_{k}$ ). 
Afirmação 1: $1 \leq y_{i}^{(k)} \leq u$.

De fato, como $p_{k}|1+k t, 1+k t| 1+c t$ e, para $i=1, \ldots, m, 1+c t \mid \prod_{j=1}^{u}\left(a_{i}-j\right)$, então para $i=1, \ldots, m, p_{k} \mid \prod_{j=1}^{u}\left(a_{i}-j\right)$ e como $p_{k}$ é primo, para cada $i=1, \ldots, m$, existe $j=1, \ldots, u$ tal que $p_{k} \mid a_{i}-j$, ou seja, $a_{i} \equiv j\left(\bmod p_{k}\right)$. Além disso, como $y_{i}^{(k)}=r\left(a_{i}, p_{k}\right)$, temos que $a_{i} \equiv y_{i}^{(k)}\left(\bmod p_{k}\right)$, e portanto para cada $i=1, \ldots, m$, existe $j=1, \ldots, u$ tal que $y_{i}^{(k)} \equiv j$ $\left(\bmod p_{k}\right)$. Afirmamos agora que $p_{k}>Q$, pois se $p_{k} \leq Q$, então $p_{k} \mid Q$ ! e como $Q !=t$, teríamos $p_{k} \mid k t$, e como $p_{k} \mid 1+k t$, resultaria $p_{k} \mid 1$, o que é impossível.

Logo, $p_{k}>Q$ e da hipótese (1), $Q>u$ e como $j \leq u$, temos portanto que $j<p_{k}$.

Por outro lado, como $y_{i}^{(k)}=r\left(a_{i}, p_{k}\right)$, então $y_{i}^{(k)}<p_{k}$. Reunimos então a seguinte situação:

$$
y_{i}^{(k)} \equiv j\left(\bmod p_{k}\right), j<p_{k} \quad \text { e } \quad y_{i}^{(k)}<p_{k} .
$$

Portanto, $y_{i}^{(k)}=j$, e como $1 \leq j \leq u$, temos que $1 \leq y_{i}^{(k)} \leq u$.

Afirmação 2: Para $k=1, \ldots, y, P\left(y, k, x_{1}, \ldots, x_{n}, y_{1}^{(k)}, \ldots, y_{m}^{(k)}\right)=0$.

De fato, como $p_{k} \mid 1+k t$ e $1+k t \mid 1+c t$, temos

$$
1+c t \equiv 1+k t \equiv 0\left(\bmod p_{k}\right)
$$

donde

$$
k+k c t \equiv c+k c t \equiv 0\left(\bmod p_{k}\right),
$$

e portanto, para $k=1, \ldots, y, k \equiv c\left(\bmod p_{k}\right)$.

Por outro lado, como $y_{i}^{(k)}=r\left(a_{i}, p_{k}\right)$, então $y_{i}^{(k)} \equiv a_{i}\left(\bmod p_{k}\right)$. Estas congruências 
implicam que

$$
P\left(y, k, x_{1}, \ldots, x_{n}, y_{1}^{(k)}, \ldots, y_{m}^{(k)}\right) \equiv P\left(y, c, x_{1}, \ldots, x_{n}, a_{1}, \ldots, a_{m}\right)\left(\bmod p_{k}\right)
$$

Isso nos leva a concluir, juntamente com a hipótese, que

$$
P\left(y, k, x_{1}, \ldots, x_{n}, y_{1}^{(k)}, \ldots, y_{m}^{(k)}\right) \equiv 0\left(\bmod p_{k}\right)
$$

Agora, como $k \leq y$ e da Afirmação $1,1 \leq y_{i}^{(k)} \leq u$, resulta da hipótese (3) que

$$
\left|P\left(y, k, x_{1}, \ldots, x_{n}, y_{1}^{(k)}, \ldots, y_{m}^{(k)}\right)\right| \leq Q\left(y, u, x_{1}, \ldots, x_{n}\right)
$$

Já vimos que $Q\left(y, u, x_{1}, \ldots, x_{n}\right)<p_{k}$. Temos então a seguinte situação:

$$
P\left(y, k, x_{1}, \ldots, x_{n}, y_{1}^{(k)}, \ldots, y_{m}^{(k)}\right) \equiv 0\left(\bmod p_{k}\right)
$$

e

$$
\left|P\left(y, k, x_{1}, \ldots, x_{n}, y_{1}^{(k)}, \ldots, y_{m}^{(k)}\right)\right|<p_{k}
$$

Então, $P\left(y, k, x_{1}, \ldots, x_{n}, y_{1}^{(k)}, \ldots, y_{m}^{(k)}\right)=0$. Portanto,

$$
(\forall k)_{\leq y}\left(\exists y_{1}, \ldots, y_{m}\right)_{\leq u}\left[P\left(y, k, x_{1}, \ldots, x_{n}, y_{1}, \ldots, y_{m}\right)=0\right]
$$

Teorema 5.3 Seja $P\left(y, k, x_{1}, \ldots, x_{n}, y_{1}, \ldots, y_{m}\right)$ um polinômio. Então os predicados

(a) $(\exists k)_{\leq y} \exists y_{1} \cdots \exists y_{m}\left[P\left(y, k, x_{1}, \ldots, x_{n}, y_{1}, \ldots, y_{m}\right)=0\right]$;

(b) $\left.(\forall k)_{\leq y} \exists y_{1} \cdots \exists y_{m} \mid P\left(y, k, x_{1}, \ldots, x_{n}, y_{1}, \ldots, y_{m}\right)=0\right]$

são diofantinos. 
Demonstração: (a) É imediato, pois basta observar que

$$
\begin{gathered}
(\exists k)_{\leq y} \exists y_{1}, \ldots, y_{m}\left[P\left(y, k, x_{1}, \ldots, x_{n}, y_{1}, \ldots, y_{m}\right)=0\right] \\
\mathbb{1} \\
\exists k \exists y_{1}, \ldots, y_{m}\left[k \leq y \wedge P\left(y, k, x_{1}, \ldots, x_{n}, y_{1}, \ldots, y_{m}\right)=0\right] .
\end{gathered}
$$

(b) Dado o polinômio $P\left(y, k, x_{1}, \ldots, x_{n}, y_{1}, \ldots, y_{m}\right)$, vamos construir um polinômio

$$
Q\left(y, u, x_{1}, \ldots, x_{n}\right)
$$

que satisfaça as condições (1), (2) e (3) do Lema 5.2 .

Primeiramente, escrevemos o polinômio dado como soma de monômios, isto é,

$$
P\left(y, k, x_{1}, \ldots, x_{n}, y_{1}, \ldots, y_{m}\right)=\sum_{r=1}^{N} t_{r},
$$

onde cada termo $t_{r}$ é da forma $t_{r}=c y^{a} k^{b} x_{1}^{q_{1}} \cdots x_{n}^{q_{r}} y_{1}^{s_{1}} \cdots y_{m}^{s_{m}}$, com $c \in \mathbb{Z}$.

Seja agora $u_{r}=|c| y^{a+b} x_{1}^{q_{1}} \cdots x_{n}^{q_{n}} u^{s_{1}+\cdots+s_{n}}$. O polinômio desejado será então

$$
Q\left(y, u, x_{1}, \ldots x_{n}\right)=u+y+\sum_{r=1}^{N} u_{r}
$$

É fácil ver que as propriedades (1), (2) e (3) do Lema 5.2 são imediatamente satisfeitas.

De posse do polinômio $Q\left(y, u, x_{1}, \ldots x_{n}\right)$, podemos agora aplicar o Lema 5.2 e em seguida o Lema 5.1 para obter a equivalência:

$$
(\forall k)_{\leq y} \exists y_{1}, \cdots, y_{m}\left[P\left(y, k, x_{1}, \ldots, x_{n}, y_{1}, \ldots, y_{m}\right)=0\right]
$$




$$
\begin{aligned}
\exists u, c, t, a_{1}, \ldots a_{m}\left[1+c t=\prod_{k=1}^{y}(1+k t) \wedge t=Q\left(y, u, x_{1}, \ldots, x_{n}\right) !\right. \\
\wedge 1+c t\left|\prod_{j=1}^{u}\left(a_{1}-j\right) \wedge \cdots \wedge 1+c t\right| \prod_{j=1}^{u}\left(a_{m}-j\right) \\
\left.\wedge P\left(y, c, x_{1}, \ldots, x_{n}, a_{1}, \ldots, a_{m}\right) \equiv 0(\bmod 1+c t)\right]
\end{aligned}
$$

$$
\begin{aligned}
& \exists u, c, t, a_{1}, \ldots, a_{m}, e, f, g_{1}, \ldots, g_{m}, h_{1}, \ldots, h_{m}, l[e=1+c t \\
& \quad \wedge e=\prod_{k=1}^{y}(1+k t) \wedge f=Q\left(y, u, x_{1}, \ldots, x_{n}\right) \wedge t=f ! \\
& \wedge g_{1}=a_{1}-u-1 \wedge g_{2}=a_{2}-u-1 \wedge \cdots \wedge g_{m}=a_{m}-u-1 \\
& \wedge h_{1}=\prod_{k=1}^{u}\left(g_{1}+k\right) \wedge h_{2}=\prod_{k=1}^{u}\left(g_{2}+k\right) \wedge \cdots \wedge h_{m}=\prod_{k=1}^{u}\left(g_{m}+k\right) \\
& \wedge e\left|h_{1} \wedge e\right| h_{2} \wedge \cdots \wedge e\left|h_{m} \wedge e\right| l \\
&\left.\wedge l=P\left(y, k, x_{1}, \ldots, x_{n}, a_{1}, \ldots, a_{m}\right)\right] .
\end{aligned}
$$

Portanto, essa equivalência mostra que o predicado

$$
(\forall k)_{\leq y} \exists y_{1}, \cdots, y_{m}\left[P\left(y, k, x_{1}, \ldots, x_{n}, y_{1}, \ldots, y_{m}\right)=0\right]
$$

é diofantino pelos teoremas anteriores. 


\section{Capítulo 6}

\section{Funções recursivas}

\section{Definição 6.1 Função recursiva}

(1) Funções iniciais

- as funções $c(x)=1$ (constante), $s(x)=x+1$ (sucessor) e $\cup_{i}^{n}\left(x_{1}, \ldots, x_{n}\right)=x_{i}$, $1 \leq i \leq n$ (projeção) são recursivas.

(2) Composição

- se $f\left(y_{1}, \ldots, y_{m}\right), g_{1}\left(x_{1}, \ldots, x_{n}\right), \ldots, g_{m}\left(x_{1}, \ldots, x_{n}\right)$ são recursivas, então a função

$$
h\left(x_{1}, \ldots, x_{n}\right)=f\left(g_{1}\left(x_{1}, \ldots, x_{n}\right), \ldots, g_{m}\left(x_{1}, \ldots, x_{n}\right)\right)
$$

é recursiva.

(3) Recursão primitiva

- se $f\left(x_{1}, \ldots, x_{n}\right)$ e $g\left(t, y, x_{1}, \ldots, x_{n}\right)$ são recursivas e $h\left(x_{1}, \ldots, x_{n}, t\right)$ é uma função 
que satisfaz a equação

$$
\left\{\begin{array}{l}
h\left(x_{1}, \ldots, x_{n}, 1\right)=f\left(x_{1}, \ldots, x_{n}\right) \\
h\left(x_{1}, \ldots, x_{n}, t+1\right)=g\left(t, h\left(x_{1}, \ldots, x_{n}, t\right), x_{1}, \ldots, x_{n}\right)
\end{array}\right.
$$

então $h\left(x_{1}, \ldots, x_{n}, t\right)$ é recursiva.

(4) Minimalização (Se $R(x)$ é uma relação tal que $\exists x R(x)$, então $\mu x R(x)$ é o menor número que satisfaz a relação $R(x)$.)

- se $f\left(x_{1}, \ldots, x_{n}, y\right)$ e $g\left(x_{1}, \ldots, x_{n}, y\right)$ são recursivas e tais que para todo $x_{1}, \ldots, x_{n}$ existe $y$ tal que $f\left(x_{1}, \ldots, x_{n}, y\right)=g\left(x_{1}, \ldots, x_{n}, y\right)$, então a função

$$
h\left(x_{1}, \ldots, x_{n}\right)=\mu y\left(f\left(x_{1}, \ldots, x_{n}, y\right)=g\left(x_{1}, \ldots, x_{n}, y\right)\right)
$$

é recursiva.

(5) As funções recursivas são obtidas unicamente pela aplicação das cláusulas (1) - (4).

Observação: Usualmente as funções recursivas são definidas sobre os números naturais, ao passo que aqui as funções recursivas são definidas excluindo-se o zero de seu domínio. Notemos contudo que uma função $f\left(x_{1}, \ldots, x_{n}\right)$ é recursiva como aqui definimos se e somente se a função $F\left(t_{1}, \ldots, t_{n}\right)=f\left(t_{1}+1, \ldots, t_{n}+1\right)-1$ é recursiva no sentido usual.

Proposição 6.2 A funçâo $S(i, u)$ e as funçôes polinomiais com coeficientes inteiros positivos sâo recursivas.

Demonstração: Como a função $S(i, u)$ e os polinômios com coeficientes inteiros positivos definidas sobre os números naturais são recursivas na definição usual, a proposição segue da observação acima. 
Teorema 6.3 Uma funçâo é diofantina se e somente se é recursiva.

Demonstração: Abreviemos $\left(x_{1}, \ldots, x_{n}\right)$ por $\vec{x}$.

$(\Rightarrow)$ Seja $f$ diofantina. Então existem polinômios $P$ e $Q$ com coeficientes inteiros tal que

$$
y=f(\vec{x}) \Leftrightarrow \exists t_{1}, \ldots, t_{m}\left(P\left(\vec{x}, y, t_{1}, \ldots, t_{m}\right)=Q\left(\vec{x}, y, t_{1}, \ldots, t_{m}\right)\right)
$$

Então,

$$
\exists t_{1}, \ldots, t_{m}\left(P\left(\vec{x}, f(\vec{x}), t_{1}, \ldots, t_{m}\right)=Q\left(\vec{x}, f(\vec{x}), t_{1}, \ldots, t_{m}\right)\right)
$$

e então pela Proposição 1.13

$$
\exists u(P(\vec{x}, S(1, u), S(2, u), \ldots, S(m+1, u))=Q(\vec{x}, S(1, u), S(2, u), \ldots, S(m+1, u))
$$

Como $S(i, u)$ e os polinômios são funçōes recursivas, usando a composição e minimalização temos que também é recursiva a função

$$
\varphi(\vec{x})=\mu u(P(\vec{x}, S(1, u), \ldots, S(m+1, u))=Q(\vec{x}, S(1, u), \ldots, S(m+1, u)) .
$$

Temos então que

$$
P(\vec{x}, S(1, \varphi(\vec{x})), \ldots, S(m+1, \varphi(\vec{x})))=Q(\vec{x}, S(1, \varphi(\vec{x})), \ldots, S(m+1, \varphi(\vec{x})))
$$

Isso implica que

$$
\exists t_{1}, \ldots, t_{m}\left(P\left(\vec{x}, S(1, \varphi(\vec{x})), t_{1}, \ldots, t_{m}\right)=Q\left(\vec{x}, S(1, \varphi(\vec{x})), t_{1}, \ldots, t_{m}\right)\right)
$$

Como $y=f(\vec{x}) \Leftrightarrow \exists t_{1}, \ldots, t_{m}\left(P\left(\vec{x}, y, t_{1}, \ldots, t_{m}\right)=Q\left(\vec{x}, y, t_{1}, \ldots, t_{m}\right)\right)$, segue então que $f(\vec{x})=S(1, \varphi(\vec{x}))$. 
Como já comentamos, $S(i, u)$ e $\varphi(\vec{x})$ são funções recursivas, logo, $f$ é recursiva. Portanto, toda função diofantina é recursiva.

$(\Leftarrow)$ Seja $f$ recursiva.

As funções iniciais $c(x)=1, S(x)=x+1$ e $\cup_{i}^{n}\left(x_{1}, \ldots, x_{n}\right)=x_{i}, 1 \leq i \leq n$, são trivialmente diofantinas.

Analisemos os esquemas da composição, recursão e minimalização.

- Caso 1: Composição

Seja $f(\vec{x})=h\left(g_{1}(\vec{x}), \ldots, g_{m}(\vec{x})\right)$, onde $h, g_{1}, \ldots, g_{m}$ são recursivas. Então

$$
y=f(\vec{x}) \Leftrightarrow \exists t_{1}, \ldots, t_{m}\left(t_{1}=g_{1}(\vec{x}) \wedge \cdots \wedge t_{m}=g_{m}(\vec{x}) \wedge y=h\left(t_{1}, \ldots, t_{m}\right)\right) .
$$

Como por hipótese de indução $g_{1}, \ldots, g_{m}, h$ são diofantinas, segue da equivalência acima que $f$ é diofantina.

- Caso 2: Recursão

Seja $\left\{\begin{array}{l}f(\vec{x}, 1)=g(\vec{x}) \\ f(\vec{x}, t+1)=h(t, f(\vec{x}, t), \vec{x})\end{array}\right.$ com $g$ e $h$ recursivas. Dado um número $z$, usamos a função $S(i, u)$ para codificar os números $f(\vec{x}, 1), \ldots, f(\vec{x}, z)$. Então

$$
\begin{aligned}
y=f(\vec{x}, z) \Leftrightarrow & \exists u[\exists v(v=S(1, u) \wedge v=g(\vec{x})) \\
& \wedge(\forall t)_{\leq z}(t=z \vee \exists v(v=S(t+1, u) \wedge v=h(t, S(t, u), \vec{x}))) \\
& \wedge y=S(z, u)]
\end{aligned}
$$

Por hipótese de indução, $g$ e $h$ são diofantinas e como predicados diofantinos são fechados para o quantificador limitado $(\forall t)_{\leq z}$, segue que $f$ é diofantina. 
- Caso 3: Minimalização

Seja $f(\vec{x})=\mu y(g(\vec{x}, y)=h(\vec{x}, y))$, onde $g$ e $h$ são recursivos. Então

$$
\begin{aligned}
y=f(\vec{x}) & \Leftrightarrow \exists u(u=g(\vec{x}, y) \wedge u=h(\vec{x}, y)) \\
& \wedge(\forall t)_{\leq y}[t=y \vee \exists z, w(z=g(\vec{x}, t) \wedge w=h(\vec{x}, t) \wedge(z<w \vee w<z))]
\end{aligned}
$$

Por hipótese de indução, $g$ e $h$ são diofantinas, e como os predicados diofantinos são fechados para o quantificador limitado $(\forall t)_{\leq y}$, segue que $f$ é diofantina.

Portanto, toda função recursiva é diofantina. 


\section{Capítulo 7}

\section{O décimo problema de Hilbert é insolúvel}

Vamos apresentar uma enumeração de todos os conjuntos diofantinos $D \subseteq \mathbb{N}^{*}$.

Fixemos $x_{0}, x_{1}, x_{2}, \ldots$ como variáveis.

A notação $\left.P\left(x_{0}, x_{1}, \ldots, x_{n}\right)\right|_{\left(t_{0}, t_{1}, \ldots, t_{n}\right)} ^{\left(x_{0}, x_{1}\right)}$ denotará o polinômio obtido a partir de $P$, pela substituição de $\left(x_{0}, x_{1}, \ldots, x_{n}\right)$ pelos termos $\left(t_{0}, t_{1}, \ldots, t_{n}\right)$. Quando não houver risco de confusão, escreveremos $\left.P\left(x_{0}, x_{1}, \ldots, x_{n}\right)\right|_{\left(t_{0}, t_{1}, \ldots, t_{n}\right)} ^{\left(x_{0}, x_{1}, \ldots, x_{n}\right)}$ simplesmente como $P\left(t_{0}, t_{1}, \ldots, t_{n}\right)$. Usaremos de forma análoga a notação $\left.P\left(x_{0}, x_{1}, \ldots, x_{n}\right)\right|_{t} ^{x_{i}}$.

- Enumeração de polinômios

Todo polinômio com coeficientes inteiros positivos pode ser obtido a partir de 1 e das variáveis por meio de adições e multiplicações sucessivas.

Apresentamos então a seguinte enumeração de polinômios com coeficientes inteiros 
positivos utilizando as funções $L(z)$ e $R(z)$ :

$$
\left\{\begin{array}{l}
P_{1}=1 \\
P_{3 i-1}=x_{i-1} \\
P_{3 i}=P_{L(i)}+P_{R(i)} \\
P_{3 i+1}=P_{L(i)} \cdot P_{R(i)}
\end{array}\right.
$$

Ao escrevermos $P_{i}=P_{i}\left(x_{0}, x_{1}, \ldots, x_{n}\right)$, estará implícito que $n$ será suficientemente grande para englobar todas as variáveis de $P_{i}$ embora obviamente nem todas as variáveis $x_{0}, x_{1}, \ldots, x_{n}$ ocorram em $P_{i}$.

Observamos ainda que em $P_{n}$ não ocorrem variáveis com índice superior a $n$. De fato, para $n=1$ é imediato. Se $n=3 i-1$, então $P_{n}=P_{3 i-1}=x_{i-1}$ e $x_{i-1}$ não é $x_{j}$, para todo $j \geq n$. Se $n=3 i$, então $P_{n}=P_{3 i}=P_{L(i)}+P_{R(i)}$ e como $L(i), R(i) \leq i<3 i=n$, por hipótese de indução não ocorrem variáveis $x_{j}$, com $j>n$, em $P_{L(i)}$ e em $P_{R(i)}$ e, portanto, em $P_{n}$. O caso $n=3 i+1$ segue de maneira análoga.

A observação acima nos mostra que na expressão

$$
\exists x_{1}, \ldots, x_{n}\left(P_{L(n)}\left(x_{0}, x_{1}, \ldots, x_{n}\right)=P_{R(n)}\left(x_{0}, x_{1}, \ldots, x_{n}\right)\right)
$$

todas as variáveis estão quantificadas existencialmente, exceto $x_{0}$ : quando esta ocorre.

Definição 7.1 Para $n \geq 1$, definimos

$$
D_{n}=\left\{x: \exists x_{1}, \ldots, x_{n}\left(\left.P_{L(n)}\left(x_{0}, x_{1}, \ldots, x_{n}\right)\right|_{x} ^{x_{0}}=\left.P_{R(n)}\left(x_{0}, x_{1}, \ldots, x_{n}\right)\right|_{x} ^{x_{0}}\right)\right\}
$$

Teorema 7.2 Para todo conjunto diofantino $S \subseteq \mathbb{N}^{*}$, existe $n \geq 1$ tal que $S=D_{n}$. 
Demonstração: Seja $S \subseteq \mathbb{N}^{*}$ diofantino. Então existe um polinômio $P\left(x, t_{1}, \ldots, t_{n}\right)$ com coeficientes inteiros tal que

$$
x \in S \leftrightarrow \exists t_{1}, \ldots, t_{n}\left(P\left(x, t_{1}, \ldots, t_{n}\right)=0\right)
$$

Podemos de maneira óbvia escrever a expressão $P\left(x, t_{1}, \ldots, t_{n}\right)=0$ como uma igualdade $Q\left(x, t_{1}, \ldots, t_{n}\right)=R\left(x, t_{1}, \ldots, t_{n}\right)$, onde $Q$ e $R$ são polinômios com coeficientes inteiros positivos. Então podemos escrever

$$
x \in S \leftrightarrow \exists t_{1}, \ldots, t_{n}\left(Q\left(x, t_{1}, \ldots, t_{n}\right)=R\left(x, t_{1}, \ldots, t_{n}\right)\right)
$$

Basta tomar então $n$ tal que $P_{L(n)}=Q$ e $P_{R(n)}=R$ e então

$$
x \in S \leftrightarrow \exists x_{1}, \ldots, x_{n}\left(P_{L(n)}\left(x, x_{1}, \ldots, x_{n}\right)=P_{R(n)}\left(x, x_{1}, \ldots, x_{n}\right)\right),
$$

ou seja, $S=D_{n}$.

Teorema 7.3 O conjunto $\left\{(n, x): x \in D_{n}\right\}$ é diofantino.

Demonstração: Vamos mostrar que

$$
\begin{aligned}
& x \in D_{n} \Leftrightarrow \exists u[S(1, u)=1 \wedge S(2, u)=x \\
& \wedge(\forall i)_{\leq n}(S(3 i, u)=S(L(i), u)+S(R(i), u)) \\
& \wedge(\forall i)_{\leq n}(S(3 i+1, u)=S(L(i), u) \cdot S(R(i), u)) \\
&\wedge S(L(n), u)=S(R(n), u)]
\end{aligned}
$$


De fato:

$(\Rightarrow)$ Suponha $x \in D_{n}$. Então existem $t_{1}, \ldots, t_{n}$ tais que

$$
P_{L(n)}\left(x, t_{1}, \ldots, t_{n}\right)=P_{R(n)}\left(x, t_{1}, \ldots, t_{n}\right)
$$

Tomemos $u$ tal que $S(j, u)=P_{j}\left(x, t_{1}, \ldots, t_{n}\right)$, para $j=1,2, \ldots, 3 n+2$. Então temos que

- $S(1, u)=P_{1}\left(x, t_{1}, \ldots, t_{n}\right)=1$;

- $S(2, u)=P_{2}\left(x, t_{1}, \ldots, t_{n}\right)=x$;

- Seja $i \leq n$. Então $3 i \leq 3 n<3 n+2$. Logo,

$$
S(3 i, u)=P_{3 i}\left(x, t_{1}, \ldots, t_{n}\right)=P_{L(i)}\left(x, t_{1}, \ldots, t_{n}\right)+P_{R(i)}\left(x, t_{1}, \ldots, t_{n}\right)
$$

e como $L(i), R(i) \leq i \leq n<3 n+2$, segue que

$$
P_{L(i)}\left(x, t_{1}, \ldots, t_{n}\right)+P_{R(i)}\left(x, t_{1}, \ldots, t_{n}\right)=S(L(i), u)+S(R(i), u) .
$$

Portanto,

$$
(\forall i)_{\leq n}(S(3 i, u)=S(L(i), u)+S(R(i), u)) ;
$$

- Notando que se $i \leq n$, então $3 i+1 \leq 3 n+1<3 n+2$, a afirmação

$$
(\forall i)_{\leq n}(S(3 i+1, u)=S(L(i), u) \cdot S(R(i), u))
$$

é justificada de forma análoga ao caso anterior;

- Finalmente, como $L(n), R(n) \leq n<3 n+2$, segue que $S(L(n), u)=P_{L(n)}\left(x, t_{1}, \ldots, t_{n}\right)$ e $S(R(n), u)=P_{R(n)}\left(x, t_{1}, \ldots, t_{n}\right)$ e como $P_{L(n)}\left(x, t_{1}, \ldots, t_{n}\right)=P_{R(n)}\left(x, t_{1}, \ldots, t_{n}\right)$, temos que $S(L(n), u)=S(R(n), u)$. 
$(\Leftarrow)$ Suponhamos agora que para dados $x, n$, a expressão do lado direito da equivalência seja válida. Para $i=1,2, \ldots, n$, façamos $t_{i}=S(3 i+2, u)$.

Afirmação: Para $j \leq 3 n+2, S(j, u)=P_{j}\left(x, t_{1}, \ldots, t_{n}\right)$.

De fato:

- $S(1, u)=1=P_{1}\left(x, t_{1}, \ldots, t_{n}\right)$;

- $S(2, u)=x=P_{2}\left(x, t_{1}, \ldots, t_{n}\right)$;

- Se $j=3 i$, então $3 i \leq 3 n+2<3 n+3=3(n+1)$. Logo, $i \leq n$. Então, por hipótese

$$
S(j, u)=S(3 i, u)=S(L(i), u)+S(R(i), u)
$$

e como $L(i), R(i) \leq i<3 i=j$, por hipótese de indução, teremos que

$$
\begin{aligned}
S(L(i), u)+S(R(i), u) & =P_{L(i)}\left(x, t_{1}, \ldots, t_{n}\right)+P_{R(i)}\left(x, t_{1}, \ldots, t_{n}\right) \\
& =P_{3 i}\left(x, t_{1}, \ldots, t_{n}\right)=P_{j}\left(x, t_{1}, \ldots, t_{n}\right) .
\end{aligned}
$$

Portanto, se $j=3 i$, então $S(j, u)=P_{j}\left(x, t_{1}, \ldots, t_{n}\right)$;

- Se $j=3 i+1$, então $3 i+1 \leq 3 n+2<3 n+4=3(n+1)+1$. logo, $i \leq n$ e usando a mesma argumentação do caso anterior, concluímos que se $j=3 i+1$, então $S(j, u)=P_{j}\left(x, t_{1}, \ldots, t_{n}\right)$;

- Se $j=3 i+2$, como $j=3 i+2 \leq 3 n+2$, então $i \leq n$, logo, por definição, $S(3 i+2, u)=t_{i}$. 
Notemos que na nossa enumeração de polinômios, $P_{3 i-1}=x_{i-1}$ é equivalente a

$$
P_{2}=x_{0} \wedge P_{3(i+1)-1}=x_{i}
$$

Já vimos que $S(2, u)=x=P_{2}\left(x, t_{1}, \ldots, t_{n}\right)$. No outro caso, teremos então, para $i=$ $1, \ldots, n$ :

$$
\begin{aligned}
S(j, u) & =S(3 i+2, u)=t_{i}=P_{3(i+1)-1}\left(x, t_{1}, \ldots, t_{n}\right) \\
& =P_{3 i+1}\left(x, t_{1}, \ldots, t_{n}\right)=P_{j}\left(x, t_{1}, \ldots, t_{n}\right) .
\end{aligned}
$$

Portanto, se $j=3 i+2$, então $S(j, u)=P_{j}\left(x, t_{1}, \ldots, t_{n}\right)$.

Isso termina a prova de que para $j \leq 3 n+2, S(j, u)=P_{j}\left(x, t_{1}, \ldots, t_{n}\right)$.

Em particular, como $L(n), R(n) \leq n<3 n+2$, temos que $S(L(n), u)=P_{L(n)}\left(x, t_{1}, \ldots, t_{n}\right)$ e $S(R(n), u)=P_{R(n)}\left(x, t_{1}, \ldots, t_{n}\right)$. Por hipótese, $S(L(n), u)=S(R(n), u)$, então

$$
P_{L(n)}\left(x, t_{1}, \ldots, t_{n}\right)=P_{R(n)}\left(x, t_{1}, \ldots, t_{n}\right),
$$

ou seja, $x \in D$.

A equivalência está demonstrada e a expressão da direita é diofantina. Portanto, o conjunto $\left\{(n, x): x \in D_{n}\right\}$ é diofantino.

Teorema 7.4 $O$ conjunto $V=\left\{n: n \notin D_{n}\right\}$ nâo é diofantino.

Demonstração: Se $V$ é diofantino, então $V=D_{i}$ para algum $i$, e nesse caso teríamos $i \in D_{i} \Leftrightarrow i \in V \Leftrightarrow i \notin D_{i}$, uma contradição. 
Observação: Os Teoremas 7.3 e 7.4 nos mostram que os predicados diofantinos não são fechado para os conectivos $\neg, \rightarrow$ e o quantificador $\forall$. De fato, pelo Teorema 7.3, existe um polinômio $Q$ tal que

$$
x \in D_{n} \Leftrightarrow \exists t_{1}, \ldots, t_{n}\left(Q\left(n, x, t_{1}, \ldots, t_{m}\right)=0\right)
$$

e portanto, lembrando que $V=\left\{n: n \notin D_{n}\right\}$, temos

$$
\begin{aligned}
x \in V & \Leftrightarrow x \notin D_{x} \Leftrightarrow \neg \exists t_{1}, \ldots, t_{m}\left(Q\left(x, x, t_{1}, \ldots, t_{m}\right)=0\right) \\
& \Leftrightarrow \exists t_{1}, \ldots, t_{m}\left(Q\left(x, x, t_{1}, \ldots, t_{m}\right)=0\right) \rightarrow 1=0 \\
& \Leftrightarrow \forall t_{1}, \ldots, t_{m}\left(Q\left(x, x, t_{1}, \ldots, t_{n}\right)>0 \vee Q\left(x, x, t_{1}, \ldots, t_{n}\right)<0\right) .
\end{aligned}
$$

Como $x \in V$ não é diofantino e os predicados

$$
\begin{aligned}
& \exists t_{1}, \ldots, t_{m}\left(Q\left(x, x, t_{1}, \ldots, t_{m}\right)=0\right), 1=0 \quad \mathrm{e} \\
& \left(Q\left(x, x, t_{1}, \ldots, t_{m}\right)>0 \vee Q\left(x, x, t_{1}, \ldots, t_{m}\right)<0\right)
\end{aligned}
$$

são diofantinos, as equivalências acima mostram que a introdução do quantificador $\forall \mathrm{e}$ dos conectivos $\neg$, $\rightarrow$ pode resultar em predicados não diofantinos.

Teorema 7.5 O predicado $x \in D_{n}$ não é recursivo.

Demonstração: Suponhamos que $x \in D_{n}$ é recursivo. Então sua função característica

$$
g(n, x)= \begin{cases}1 & \text { se } x \notin D_{n} \\ 2 & \text { se } x \in D_{n}\end{cases}
$$

é recursiva e, portanto, pelo Teorema $6.3, g(n, x)$ é uma função diofantina. 
Então existe um polinômio $P\left(n, x, y, t_{1}, \ldots, t_{n}\right)$ tal que

$$
g(n, x)=y \Leftrightarrow \exists t_{1}, \ldots, t_{n}\left(P\left(n, x, y, t_{1}, \ldots, t_{m}\right)=0\right)
$$

e portanto

$$
g(n, n)=1 \Leftrightarrow \exists t_{1}, \ldots, t_{m}\left(P\left(n, n, 1, t_{1}, \ldots, t_{m}\right)=0\right)
$$

e como $g(n, n)=1 \Leftrightarrow n \notin D_{n}$, segue que

$$
n \notin D_{n} \Leftrightarrow \exists t_{1}, \ldots, t_{m}\left(P\left(n, n, 1, t_{1}, \ldots, t_{m}\right)=0\right)
$$

e isso mostra que $n \notin D_{n}$ é um predicado diofantino, contra o Teorema 7.4.

Teorema 7.6 (O décimo problema de Hilbert é insolúvel.)

Nâo existe um algoritmo que decida se uma equaçào polinomial qualquer $P\left(x_{1}, \ldots, x_{n}\right)=$ 0 admite soluções.

Demonstração: Suponhamos que um tal algoritmo $H$ exista. Pelo Teorema 7.3, o predicado $x \in D_{n}$ é diofantino, ou seja, existe um polinômio $Q\left(n, x, y, t_{1}, \ldots, t_{n}\right)$ tal que

$$
x \in D_{n} \Leftrightarrow \exists t_{1}, \ldots, t_{m}\left(Q\left(n, x, t_{1}, \ldots, t_{m}\right)=0\right)
$$

Então, para cada $x, n$, poderíamos utilizar o algoritmo $H$ para verificar se existe ou não solução para a equação $Q\left(n, x, t_{1}, \ldots, t_{m}\right)=0$. A expressão acima mostra que se existe solução, então $x \in D_{n}$, caso contrário, $x \notin D_{n}$, e portanto o predicado $x \in D_{n}$ seria recursivo, contra o Teorema 7.5.

Portanto, não existe tal algoritmo e o décimo problema de Hilbert é insolúvel. 


\section{Capítulo 8}

\section{Conjuntos recursivamente enumeráveis, grau e dimensão}

Definição 8.1 Um conjunto $R \subseteq \mathbb{N}_{*}^{n}$ é recursivamente enumerável (r.e.) se e somente se existem funções recursivas $f$ e $g$ tais que

$$
R=\left\{\left(x_{1}, \ldots, x_{n}\right): \exists x\left(f\left(x, x_{1}, \ldots, x_{n}\right)=g\left(x, x_{1}, \ldots, x_{n}\right)\right)\right\} .
$$

Teorema 8.2 Um conjunto é diofantino se e somente se é r.e.

Demonstração: $(\Rightarrow)$ Seja $R \subseteq \mathbb{N}_{*}^{n}$ diofantino. Então existem polinômios $P$ e $Q$ tais que

$$
\left(x_{1}, \ldots, x_{n}\right) \in R \Leftrightarrow \exists t_{1}, \ldots, t_{m}\left(P\left(x_{1}, \ldots, x_{n}, t_{1}, \ldots, t_{m}\right)=Q\left(x_{1}, \ldots, x_{n}, t_{1}, \ldots, t_{m}\right)\right) .
$$

Utilizando a função $S(i, u)$ temos então que

$$
\begin{aligned}
\left(x_{1}, \ldots, x_{n}\right) \in R & \Leftrightarrow \exists u\left(P\left(x_{1}, \ldots, x_{n}, S(1, u), \ldots, S(m, u)\right)\right. \\
& \left.=Q\left(x_{1}, \ldots, x_{n}, S(1, u), \ldots, S(m, u)\right)\right) .
\end{aligned}
$$


Como $P, Q$ e $S$ são funções recursivas, segue que $R$ é r.e.

$(\Leftarrow)$ Seja $R \subseteq \mathbb{N}_{*}^{n}$ r.e. Então existem funções recursivas $f$ e $g$ tais que

$$
\begin{aligned}
\left(x_{1}, \ldots, x_{n}\right) \in R & \Leftrightarrow \exists x\left(f\left(x, x_{1}, \ldots, x_{n}\right)=g\left(x, x_{1}, \ldots, x_{n}\right)\right) \\
& \Leftrightarrow \exists x \exists y\left(y=f\left(x, x_{1}, \ldots, x_{n}\right) \wedge y=g\left(x, x_{1}, \ldots, x_{n}\right)\right) .
\end{aligned}
$$

Como $f$ e $g$ são recursivas, pelo Teorema 6.3 , os predicados

$$
y=f\left(x, x_{1}, \ldots, x_{n}\right) \quad \text { e } \quad y=g\left(x, x_{1}, \ldots, x_{n}\right)
$$

são diofantinos. Portanto, $R$ é diofantino.

Definição 8.3 Seja $S \subseteq \mathbb{N}^{*}$ um conjunto diofantino. A dimensão de $S$ é o menor número $n$ para o qual existe um polinômio $P$ tal que

$$
x \in S \Leftrightarrow \exists t_{1}, \ldots, t_{n}\left(P\left(x, t_{1}, \ldots, t_{n}\right)=0\right)
$$

Definição 8.4 Seja $S \subseteq \mathbb{N}^{*}$ um conjunto diofantino. O grau de $S$ é o menor grau de um polinômio $P$ tal que

$$
x \in S \Leftrightarrow \exists t_{1}, \ldots, t_{n}\left(P\left(x, t_{1}, \ldots, t_{n}\right)=0\right) .
$$

Observamos que o grau e a dimensão de um conjunto $S$ são conceitos a princípio independentes.

Teorema 8.5 Todo conjunto diofantino tem grau $n \leq 4$. 
Demonstração: Seja $S$ um conjunto diofantino e $P$ um polinômio tal que

$$
x \in S \Leftrightarrow \exists t_{1}, \ldots, t_{n}\left(P\left(x, t_{1}, \ldots, t_{n}\right)=0\right) .
$$

Podemos então reduzir o grau de $P$ introduzindo novas variáveis $z_{j}$ satisfazendo equações da forma $z_{j}=x^{2}, z_{j}=x t_{i}, z_{j}=t_{i}^{2}, z_{j}=t_{i} t_{k}$ e continuar o processo até obtermos equações de grau 2. Somando então os quadrados obtemos polinômio de grau 4.

Exemplo: Consideremos a equação $x^{4} y^{3} w+y^{2} z=0$. Então

$$
\begin{aligned}
& x^{4} y^{3} w+y^{2} z=0 \Leftrightarrow a=x^{2} \wedge b=y^{2} \wedge a^{2} b y w+b z=0 \\
& \Leftrightarrow a=x^{2} \wedge b=y^{2} \wedge c=a^{2} \wedge c b y w+b z=0 \\
& \Leftrightarrow a=x^{2} \wedge b=y^{2} \wedge c=a^{2} \wedge d=c b \wedge e=y w \wedge f=b z \wedge d e+f=0 \\
& \Leftrightarrow x^{2}-a=0 \wedge y^{2}-b=0 \wedge a^{2}-c=0 \wedge c b-d=0 \wedge y w-e=0 \\
& \quad \wedge b z-f=0 \wedge d e+f=0
\end{aligned}
$$

Obtivemos equações polinomiais de grau $\leq 2$. Basta agora somar os quadrados de cada polinômio e obteremos uma equação polinomial de grau 4.

Teorema 8.6 Existe um número $m$ tal que todo conjunto diofantino tem dimensão menor ou igual a $\mathrm{m}$.

Demonstração: Já vimos que para todo conjunto diofantino $S$ existe um número $n$ tal que $S=D_{n}$, e pelo Teorema 7.3, existe um polinômio $Q$ tal que

$$
x \in D_{n} \Leftrightarrow \exists t_{1}, \ldots, t_{m}\left(Q\left(x, n, t_{1}, \ldots, t_{m}\right)=0\right) .
$$


Portanto, a dimensão de $S$ é menor ou igual a $m$.

Observação: Podemos construir o polinômio $Q$ do Teorema 7.3 pelos métodos aqui expostos e chegaremos então a um polinômio cuja dimensão é próxima a 50. No entanto, é possível reduzir bastante tal dimensão. 


\section{Capítulo 9}

\section{Relações com complexidade de algoritmos}

Neste capítulo, mostraremos as relações entre a prova da insolubilidade do décimo problema de Hilbert (que doravante chamaremos de MRDP em homenagem a Matyasevic, J. Robinson, M. Davis e H. Putnam) e alguns problemas de complexidade de algoritmos. Faremos inicialmente uma breve exposição de alguns conceitos fundamentais de complexidade de algoritmos e as definições básicas de fragmentos de aritmética [3]. Passaremos depois para o teorema de Parikh [9], e com o auxílio de um teorema de Manders e Adleman [7], chegaremos finalmente ao resultado obtido por Wilkie [11], a saber, que se MRDP puder ser provado no fragmento de aritmética $I \Sigma_{0}$, então $\mathbf{N P}=c o \mathbf{N P}$. 


\subsection{Definições básicas}

\subsubsection{Linguagens formais e máquinas de Turing}

O modelo computacional adotado para a análise de complexidade de algoritmos serão as máquinas de Turing determinísticas e não determinísticas. A seguir, descreveremos de forma sucinta o modelo aqui utilizado.

Um conjunto $\Sigma$ finito e não vazio de símbolos será denominado alfabeto.

$O$ conjunto de todas as seqüências finitas de símbolos de $\Sigma$ será denotado por $\Sigma^{*}$, e um elemento $x \in \Sigma^{*}$ será chamado de palavra.

Se $x$ é uma palavra, $|x|$ denotará o número de símbolos que compõe $x$.

\subsubsection{Máquinas de Turing determinísticas (DTM) e a classe $\mathbf{P}$}

Uma máquina de Turing deterministica (DTM) consiste de uma fita infinita dividida em células, que pode mover-se para a direita ou para a esquerda, e por um cabeçote que permite ler e imprimir símbolos na célula sobre a qual se encontra.

Consideremos os seguintes conjuntos:

$$
\begin{aligned}
& \Sigma=\left\{s_{1}, s_{2}, \ldots, s_{n}\right\} \text { será o alfabeto; } \\
& Q=\left\{q_{1}, q_{2}, \ldots, q_{k}\right\} \text { o conjunto de estados internos, e }
\end{aligned}
$$


$S=\{L, H, R\}$ os comandos que indicam se o cabeçote de leitura deve permanecer parado $(H)$, ir para a esquerda $(L)$, ou para a direita $(R)$.

Dentre os elementos de $Q$, três serão destacados: $q_{0}$ (estado inicial), $q_{a}$ (estado final aceita) e $q_{r}$ (estado final rejeita)

Definição 9.1 O programa de uma máquina de Turing determinística (DTM) é um conjunto de quádruplas de uma das seguinte formas:

$\begin{array}{llll}q_{i} & s_{j} & s_{k} & q_{l} \\ q_{i} & s_{j} & L & q_{l} \\ q_{i} & s_{j} & R & q_{l} \\ q_{i} & s_{j} & H & q_{l}\end{array}$

e que satisfaça a seguinte condição de consistência: duas quádruplas distintas não podem iniciar com o mesmo par $q_{i} s_{j}$.

Vamos omitir maiores detalhes sobre os aspectos funcionais das máquinas de Turing. Omitiremos também algumas definições usuais como por exemplo de configuração e computação, pois são comuns na literatura.

Definição 9.2 Dizemos que uma DTM $T$ com entrada $x$ pára se a computação atingir um dos estados finais $q_{a}$ ou $q_{r}$, e que $T$ aceita uma palavra $x$ se e somente se $T$ com entrada $x$ pára no estado final $q_{a}$.

Se $x$ é uma palavra aceita por $T, \theta_{T}(x)$ denotará o número de passos executados por $T$ até a parada. 
Definição 9.3 Seja $\Sigma$ um alfabeto. Uma linguagem sobre o alfabeto $\Sigma$ é um conjunto $L \subseteq \Sigma^{*}$

Definição 9.4 Uma DTM T reconhece uma linguagem $L$ se para todo $x \in \Sigma^{*}$,

$$
x \in L \Leftrightarrow T \text { aceita } x
$$

A linguagem reconhecida por $T$ será denotada por $L(T)$.

Dizemos também que uma linguagem $L$ é reconhecível se existe uma DTM que reconhece $L$.

Definição 9.5 Uma DTM $T$ decide uma linguagem $L$ se para todo $x \in \Sigma^{*}$, ocorrer:

$$
\begin{aligned}
& \text { se } x \in L \text {, então } T \text { pára em } q_{a} \text { para a entrada } x \text {; } \\
& \text { se } x \notin L \text {, então } T \text { pára em } q_{r} \text { para a entrada } x .
\end{aligned}
$$

Dizemos também que uma linguagem $L$ é decidível se existe uma DTM $T$ que decide $L$, e nesse caso, dizemos que $T$ decide $L$.

Definição 9.6 Uma linguagem $L$ é decidível em tempo polinomial (indicamos $L \in \mathbf{P}$ ) se existe um polinômio $p(n)$ com coeficientes positivos e uma DTM $T$ que decide $L$ tal que para toda palavra $x$ aceita por $T$ ', $\theta_{T}(x) \leq p(|x|)$ (lembremos que $\theta_{T}(x)$ é o número de passos executados por $T$ até a parada, para uma entrada aceita $x$ ). 


\subsubsection{Máquinas não-determinísticas e a classe NP}

As máquinas de Turing não-determinísticas (NDTM) são definidas da mesma maneira que as máquinas determinísticas, exceto pelo fato de não satisfazerem necessariamente a condição de consistência. Assim, toda DTM é também uma NDTM.

A computação não-determinística se processa da mesma forma que a determinística, até ocorrer (se ocorrer) a seguinte situação a que chamaremos de nó:

se em certo passo de uma computação a NDTM se encontra no estado $q_{i}$ e o cabeçote lê o símbolo $s_{j}$, e além disso existirem duas (ou mais) quádruplas distintas que se iniciam pelo mesmo par $q_{i} s_{j}$, então o curso da computação se bifurca, dando origem a duas (ou mais) computações distintas e independentes. O processo se repete quando ocorrer novamente a mesma situação.

Para uma entrada $x$, a cada uma dessas computações paralelas daremos o nome de ramo da computação não-determinística.

Definição 9.7 Dizemos que uma NDTM pára se um de seus ramos atinge um dos estados finais $q_{a}$ ou $q_{r}$, e que uma NDTM aceita uma palavra $x$ se um de seus ramos atinge o estado final $q_{a}$.

O menor número de passos executados por uma NDTM $T$ com uma entrada aceita $x$ até a parada será denotado por $\theta_{T}(x)$ (a distinção com o caso determinístico ficará clara pelo contexto). 
Definição 9.8 Dizemos que uma NDTM $T$ reconhece uma linguagem $L$ se para todo $x \in \Sigma^{*}: x \in L \leftrightarrow T$ aceita $x$

Definição 9.9 Uma linguagem $L$ é verificável em tempo polinomial (indicamos $L \in \mathrm{NP}$ ) se existe um polinômio $p(n)$ com coeficientes positivos e uma NDTM $T$ que aceita $L$ tal que se $x \in L$, então $\theta_{T}(x) \leq p(|x|)$.

Alternativamente, podemos também caracterizar a classe NP da seguinte forma: $L \in \mathrm{NP}$ se e somente se existe um polinômio $p(x)$ e uma linguagem $Q \in \mathrm{P}$ tal que

$$
x \in L \leftrightarrow \exists y(|y| \leq p(|x|) \text { e }(x, y) \in Q)
$$

Intuitivamente, uma linguagem $L \in \mathrm{NP}$ se a afirmação $x \in L$ puder ser verificada em tempo polinomial.

Para ilustrar, tomemos como exemplo o problema SAT: "Dada uma fórmula do cálculo proposicional, existe uma valoração que a torne verdadeira?".

Sabemos que pelo método das tabelas de verdade, a resposta a esse problema é obtida após um tempo exponencial em relação ao número de letras proposicionais presentes na fórmula, tempo este que é gasto fundamentalmente na busca de uma valoração que satisfaça a fórmula dada. No entanto, se for afirmado que a fórmula é satisfatível e for dada uma valoração que a satisfaça, podemos verificar rapidamente a veracidade dessa afirmação. Essa é a noção que o conceito de verificabilidade polinomial pretende capturar. O exemplo também nos mostra que na discussão acima, não podemos trocar o sim pelo 
não, ou seja, aparentemente a verificação de que uma fórmula é satisfatível é mais rápida do que a verificação de que não é satisfatível. Isso nos motiva a definir:

Definição 9.10 Seja $\Sigma$ um alfabeto. Então coNP $=\left\{\Sigma^{*} \backslash L: L \in \mathbf{N P}\right\}$.

\subsubsection{NP-completude}

Definição 9.11 Sejam $\Sigma_{1}$ e $\Sigma_{2}$ alfabetos. Uma função $f: \Sigma_{1}^{*} \rightarrow \Sigma_{2}^{*}$ é computável em tempo polinomial se existe uma DTM $T$ que computa $f$ e um polinômio $p(n)$ com coeficientes positivos tal que para cada entrada $x, \theta_{T}(x) \leq p(|x|)$.

Definição 9.12 Sejam $\Sigma_{1}$ e $\Sigma_{2}$ alfabetos, e $L_{1} \subseteq \Sigma_{1}^{*}$ e $L_{2} \subseteq \Sigma_{2}^{*}$ linguagens. Dizemos que $L_{1}$ é redutível à $L_{2}$ em tempo polinomial $\left(L_{1} \leq{ }_{p} L_{2}\right)$ se existe uma função $f: \Sigma_{1}^{*} \rightarrow \Sigma_{2}^{*}$ computável em tempo polinomial tal que $x \in L_{1} \leftrightarrow f(x) \in L_{2}$.

Definição 9.13 Uma linguagem $L$ é NP-completa se $L \in \mathrm{NP}$ e para toda linguagem $Q \in \mathbf{N P}, Q \leq_{p} L$

Analogamente:

Definição 9.14 Uma linguagem $L$ é coNP-Completa se $L \in$ coNP e para toda linguagem $Q \in \operatorname{coNP}, Q \leq_{p} L$. 
O problema SAT a que já nos referimos, é um problema NP-completo (teorema de Cook).

O maior problema aberto da teoria da complexidade de algoritmos é saber se $\mathbf{P}=\mathbf{N P}$.

A inclusão $\mathbf{P} \subseteq \mathbf{N P}$ é imediata, pois toda DTM é também uma NDTM. Como comentamos, a inclusão contrária permanece em aberto, a despeito de algumas evidências de que a inclusão seja própria.

Aqui, esboçaremos um resultado mostrando que se admitirmos certas hipóteses, então $\mathrm{NP}=\operatorname{coNP}$.

\subsubsection{Fragmentos de aritmética}

Um fragmento de aritmética é um subsistema da aritmética de Peano obtido por meio de certas restrições ao esquema da indução.

Consideremos a linguagem $\mathcal{L}=\{0,1,+, \cdot, \leq\}$

Definição 9.15 Um quantificador limitado é da forma $\forall x \leq t$ (universal) ou $\exists x \leq t$ (existencial), onde $t$ é um termo que não contém $x$.

As interpretações de $\forall x \leq t \varphi$ e $\exists x \leq t \varphi$ são, respectivamente, as interpretações usuais de $\forall x(x \leq t \rightarrow \varphi)$ e $\exists x(x \leq t \wedge \varphi)$.

Definição 9.16 Uma fórmula é limitada se todos os seus quantificadores são limitados. 
Vamos definir indutivamente os conjuntos $\Sigma_{n}$ e $\prod_{n}$ :

$$
\begin{aligned}
& \Sigma_{0}=\prod_{0}=\text { conjunto das fórmulas limitadas; } \\
& \Sigma_{n+1}=\left\{\exists x_{1} \ldots \exists x_{k} \varphi: \varphi \in \prod_{n}\right\} \\
& \prod_{n+1}=\left\{\forall x_{1} \ldots \forall x_{k} \varphi: \varphi \in \Sigma_{n}\right\}
\end{aligned}
$$

(a cadeia de quantificadores antes da fórmula $\varphi$ ser vazia).

Definição $9.17 I \Sigma_{n}$ (indução sobre as $\Sigma_{n}$-fórmulas) é o conjunto consistindo do fecho universal de todas as fórmulas do tipo

$$
\varphi(0) \wedge \forall x(\varphi(x) \rightarrow \varphi(x+1)) \rightarrow \forall x \varphi(x)
$$

onde $\varphi \in \Sigma_{n}$.

\subsubsection{O sistema $P^{-}$}

O sistema $P^{-}$consiste dos axiomas de Peano sem o esquema da indução, que vamos expor a seguir:

1) $(x+y)+z=x+(y+z)$

2) $x+y=y+x$

3) $x+z=y+z \rightarrow x=y$ 
4) $x+0=x$

5) $x+1 \neq 0$

6) $(x \cdot y) \cdot z=x \cdot(y \cdot z)$

7) $x \cdot y=y \cdot x$

8) $x .1=x$

9) $x \cdot(y+z)=x \cdot y+x \cdot z$

10) $x<y<z \rightarrow x<z$

11) $(x<y) \vee(y<x) \vee(x=y)$

12) $x<y \rightarrow x+z<y+z$

13) $x<x+(y+1)$

14) $x<y \rightarrow \exists z(x+z+1=y)$

Notação: indicaremos por $P^{-}+I \Sigma_{n}$ o sistema que consiste dos axiomas de $P^{-}$e das fórmulas de $I \Sigma_{n}$.

Para os resultados que se seguem, se $T$ for uma teoria, $T^{\prime}$ é a teoria obtida a partir de $T$ adicionando-se novas constantes, mas sem nenhum novo axioma não lógico. 


\subsection{O teorema de Parikh}

Lema 9.18 Para todo $n>0$, se

$$
P^{-} \vdash \exists y\left(y \leq t_{1} \wedge \varphi(y)\right) \vee \cdots \vee \exists y\left(y \leq t_{n} \wedge \varphi(y)\right)
$$

então $P^{-} \vdash \exists y\left(y \leq t_{1}+\cdots+t_{n} \wedge \varphi(y)\right)$.

Demonstração: Por indução sobre $n$. Para $n=1$ é imediato. No passo indutivo, para simplificar a notação, vamos supor que

$$
P^{-} \vdash \exists y(y \leq a \wedge \varphi(y)) \vee \exists y(y \leq b \wedge \varphi(y))
$$

e mostrar então que $P^{-} \vdash \exists y(y \leq a+b \wedge \varphi(y))$.

De fato, como

$$
P^{-} \vdash y \leq a \wedge \varphi(y) \rightarrow(y \leq a \vee y \leq b) \wedge \varphi(y)
$$

então

$$
P^{-} \vdash \exists y(y \leq a \wedge \varphi(y)) \rightarrow \exists y[(y \leq a \vee y \leq b) \wedge \varphi(y)]
$$

Analogamente, como

$$
P^{-} \vdash y \leq b \wedge \varphi(y) \rightarrow(y \leq a \vee y \leq b) \wedge \varphi(y)
$$

então

$$
P^{-} \vdash \exists y(y \leq b \wedge \varphi(y)) \rightarrow \exists y[(y \leq a \vee y \leq b) \wedge \varphi(y)]
$$

De (I) e (II) obtemos

$$
P^{-} \vdash \exists y(y \leq a \wedge \varphi(y)) \vee \exists y(y \leq b \wedge \varphi(y)) \rightarrow \exists y[(y \leq a \vee y \leq b) \wedge \varphi(y)]
$$


Da hipótese

$$
P^{-} \vdash \exists y(y \leq a \wedge \varphi(y)) \vee \exists y(y \leq b \wedge \varphi(y))
$$

juntamente com (III), obtemos:

$$
P^{-} \vdash \exists y[(y \leq a \vee y \leq b) \wedge \varphi(y)]
$$

Por outro lado, como

$$
P^{-} \vdash y \leq a \rightarrow y \leq a+b \quad \text { e } \quad P^{-} \vdash y \leq b \rightarrow y \leq a+b,
$$

teremos então que

$$
P^{-} \vdash y \leq a \vee y \leq b \rightarrow y \leq a+b .
$$

e isso implica

$$
P^{-} \vdash(y \leq a \vee y \leq b) \wedge \varphi(y) \rightarrow(y \leq a+b) \wedge \varphi(y)
$$

e portanto

$$
P^{-} \vdash \exists y[(y \leq a \vee y \leq b) \wedge \varphi(y)] \rightarrow \exists y(y \leq a+b \wedge \varphi(y))
$$

De (IV) e (V) obtemos então

$$
P^{-} \vdash \exists y(y \leq a+b \wedge \varphi(y)) .
$$

\section{Lema 9.19 Seja}

$$
\Gamma=\left\{\forall x[\varphi(0) \wedge \forall y<x(\varphi(y) \rightarrow \varphi(y+1)) \rightarrow \varphi(x)]: \varphi \in \Sigma_{0}\right\}
$$

e $m \models P^{-}$. Então $m \models \Gamma \Leftrightarrow m \models I \Sigma_{0}$. 
Demonstração: $(\Rightarrow)$

Suponhamos que $m \vDash \varphi(0)$ e $m \models \forall y(\varphi(y) \rightarrow \varphi(y+1))$. Devemos mostrar que $m \models \forall x \varphi(x)$.

Seja $a \in m$. Tomemos $b \in m$ tal que $m \models b<a$ e $m \models \varphi(b)$. Como por hipótese $m \models \forall y(\varphi(y) \rightarrow \varphi(y+1))$, segue que $m \models \varphi(b+1)$. Portanto,

$$
m \models \forall y<a(\varphi(y) \rightarrow \varphi(y+1))
$$

Como $m \vDash \varphi(0)$ e $m \models \Gamma$, teremos que $m \vDash \varphi(a)$. Portanto, $m \vDash \forall x \varphi(x)$. Isso mostra que $m \vDash I \Sigma_{0}$.

$(\Leftarrow)$ Suponhamos que $m \vDash I \Sigma_{0}$ mas $m \nvdash \models$. Se $m \nvdash \Gamma$, então existem uma fórmula $\varphi \in \Sigma_{0}$ e um elemento $a \in m$ tal que $m \models \varphi(0), m \vDash \forall y<a(\varphi(y) \rightarrow \varphi(y+1))$ mas $m \not \models \varphi(a)$.

Afirmação 1: Existe $b \in m$ tal que

$$
m \vDash \forall y<b \varphi(y) \text { e } \forall c \in m(m \models b<c \Rightarrow m \not \forall \forall y<c \varphi(y)) .
$$

De fato, suponhamos que não, ou seja, para todo $b \in m$ :

$$
m \vDash \forall y<b \varphi(y) \Rightarrow \exists c \in m(m \models b<c \quad \text { e } \quad m \models \forall y<c \varphi(y))
$$

Consideremos $\psi(x) \equiv \forall y<x \varphi(y)$. Vamos mostrar usando $m \models I \Sigma_{0}$, que $m \models \forall x v(x)$, o que nos levará a uma contradição.

Trivialmente, temos $m \models \psi(0)$. Suponha que $y_{0} \in m$ é tal que $m \vDash \psi\left(y_{0}\right)$, ou seja, $m \models \forall y<y_{0} \varphi(y)$. Então, por $(*)$, existe $x_{0} \in m$ tal que $m \models y_{0}<x_{0}$ e $m \models \forall y<x_{0} \varphi(y)$. 
Mostremos que $m \models \forall y<y_{0}+1 \varphi(y)$. De fato, seja em $m, y<y_{0}+1$. Como $y_{0}<x_{0}$, então $y_{0}+1 \leq x_{0}$, logo, $y<x_{0}$, e como $m \vDash \forall y<x_{0} \varphi(y)$, então $m \vDash \varphi(y)$. Logo, $m \vDash \forall y<y_{0}+1 \varphi(y)$, isto é, $m \vDash \psi\left(y_{0}+1\right)$. Portanto,

$$
m \vDash \forall y(\psi(y) \rightarrow \psi(y+1)) .
$$

Temos então a seguinte situação:

$$
m \vDash \psi(0) \quad \text { e } \quad m \vDash \forall y(\psi(y) \rightarrow \psi(y+1)),
$$

e

$$
\psi(x) \equiv \forall y<x \varphi(y) \in \Sigma_{0}
$$

pois $\varphi \in \Sigma_{0}$. Como $m \models I \Sigma_{0}$, temos então que $m \models \forall x \psi(x)$, ou seja,

$$
m \models \forall x(\forall y<x \varphi(y))
$$

e isso implica que $m \models \forall x \varphi(x)$, pois dado $z_{0} \in m$, como $m \models \forall x(\forall y<x \varphi(y))$, então $m \models \forall y<z_{0}+1 \varphi(y)$, logo, $m \models \varphi\left(z_{0}\right)$. Portanto, de fato, $m \models \forall x \varphi(x)$, contra $m \nvdash \varphi(a)$. Absurdo! Isso prova a Afirmação 1.

Seja então $b \in m$ tal que

(1) $m \models \forall y<b \varphi(y)$

e

(2) $\forall c \in m(m \models b<c \Rightarrow m \forall \forall \forall<c \varphi(y))$.

Afirmação 2: $m \not \models \varphi(b)$. 
De fato, se $m \models \varphi(b)$, como de (1) temos $m \vDash \forall y<b \varphi(y)$, então $m \models \forall y<b+1 \varphi(y)$. Por outro lado, por (2), temos que $m \not \forall \forall y<b+1 \varphi(y)$, uma contradição.

Portanto, $m \not \models \varphi(b)$, e isso prova a Afirmação 2.

Afirmação 3: $0<b \leq a$.

De fato, $b \neq 0$ pela Afirmação 2 .

Por outro lado, se $a<b$, por (1) teríamos $m \vDash \varphi(a)$, mas supomos $m \forall \forall \varphi(a)$. Portanto $b \leq a$. Isso prova a Afirmação 3 .

Como $0<b \leq a$, então $0 \leq b-1<a$ e, por (1), temos que $m \models \varphi(b-1)$. Reunimos então a seguinte situação:

$$
0 \leq b-1<a, \quad m \vDash \varphi(b-1) \text { e } m \not \models \varphi(b),
$$

contra a suposição inicial $m \models \forall y<a(\varphi(y) \rightarrow \varphi(y+1))$. Absurdo! Isso mostra que $m \models \Gamma$. Portanto,

$$
m \models \Gamma \Leftrightarrow m \vDash I \Sigma_{0}
$$

Teorema 9.20 (Parikh) Seja $\varphi(x, y) \in \Sigma_{0}$. Se $P^{-}+I \Sigma_{0} \vdash \forall x \exists y \varphi(x, y)$, então existe um termo $t(x)$ de $\mathcal{L}=\{0,1,+, \cdot, \leq\}$ tal que

$$
P^{-}+I \Sigma_{0} \vdash \forall x \exists y \leq t(x) \varphi(x, y)
$$

Demonstração: Suponhamos que não, ou seja, para todo termo $t(x) \in \mathcal{L}$ :

$$
P^{-}+I \Sigma_{0} \not \forall \forall x \exists y \leq t(x) \varphi(x, y) .
$$


Seja $c$ uma nova constante.

Afirmação 1: Para todo $t(x)$ de $\mathcal{L}$ :

$$
\left(P^{-}+I \Sigma_{0}\right)^{\prime} \forall \exists y \leq t(c) \varphi(c, y)
$$

De fato, se existe um termo $t(x)$ de $\mathcal{L}$ tal que $\left(P^{-}+I \Sigma_{0}\right)^{\prime} \vdash \exists y \leq t(c) \varphi(c, y)$, então como $c$ é uma nova constante, temos que $P^{-}+I \Sigma_{0} \vdash \exists y \leq t(x) \varphi(x, y)$, de onde, por generalização, obtemos $P^{-}+I \Sigma_{0} \vdash \forall x \exists y \leq t(x) \varphi(x, y)$ contra $(*)$. Isso prova a Afirmação 1 .

Afirmação 2: Existe uma estrutura $m$ tal que

$$
m \models\left(P^{-}+I \Delta_{0}\right)^{\prime} \sqcup\{\forall y \leq t(c) \neg \varphi(c, y): t(x) \text { é termo de } \mathcal{L}\} .
$$

De fato, seja $\left(t_{i}(x)\right)_{i \in \mathbb{N}}$ uma enumeração de todos os termos de $\mathcal{L}$ que contém no máximo a variável $x$. Mostremos primeiramente que $\forall k \geq 0$, o conjunto

$$
\left(P^{-}+I \Sigma_{0}\right)^{\prime} \sqcup\left\{\forall y \leq t_{i}(c) \neg \varphi(c, y): i \leq k\right\}
$$

é consistente.

De fato, suponhamos que não. Seja então $k \geq 0$ tal que

$$
\left(P^{-}+I \Sigma_{0}\right)^{\prime} \sqcup\left\{\forall y \leq t_{i}(c) \neg \varphi(c, y): i \leq k\right\}
$$

é inconsistente. Então,

$$
\left(P^{-}+I \Sigma_{0}\right)^{\prime} \sqcup\left\{\forall y \leq t_{0}(c) \neg \varphi(c, y) \wedge \cdots \wedge \forall y \leq t_{k}(c) \neg \varphi(c, y)\right\}
$$

é inconsistente, e portanto

$$
\left(P^{-}+I \Sigma_{0}\right)^{\prime} \vdash \neg\left(\forall y \leq t_{0}(c) \neg \varphi(c, y) \wedge \cdots \wedge \forall y \leq t_{k}(c) \neg \varphi(c, y)\right)
$$


ou seja,

$$
\left(P^{-}+I \Sigma_{0}\right)^{\prime} \vdash \exists y \leq t_{0}(c) \varphi(c, y) \vee \cdots \vee \exists y \leq t_{k}(c) \varphi(c, y)
$$

de onde, pelo Lema 9.18, obtemos

$$
\left(P^{-}+I \Sigma_{0}\right)^{\prime} \vdash \exists y \leq\left(t_{0}(c)+\cdots+t_{k}(c)\right) \varphi(c, y)
$$

contra a Afirmação 1.

Isso mostra que $\forall k \geq 0$, o conjunto

$$
\left(P^{-}+I \Sigma_{0}\right)^{\prime} \sqcup\left\{\forall y \leq t_{i}(c) \neg \varphi(c, y): i \leq k\right\}
$$

é consistente e, portanto, pelo teorema da completude, admite modelo. Segue pelo teorema da compacidade que o conjunto de sentenças

$$
\left(P^{-}+I \Sigma_{0}\right)^{\prime} \sqcup\{\forall y \leq t(c) \neg \varphi(c, y): t(x) \text { é termo de } \mathcal{L}\}
$$

também admite modelo. Isso prova a Afirmação 2.

Seja então $m$ dado pela Afirmação 2 tal que

$$
m \models\left(P^{-}+I \Sigma_{0}\right)^{\prime} \sqcup\{\forall y \leq t(c) \neg \varphi(c, y): t(x) \text { é termo de } \mathcal{L}\}
$$

e consideremos o seguinte conjunto:

$$
\eta=\{a \in m: m \vDash a \leq t(c), \text { para algum termo } t(x) \text { de } \mathcal{L}\}
$$

Afirmação 3: A seguinte definição é uma interpretação de $\mathcal{L}^{\prime}=\{0,1, c,+, \cdot \leq\}$ em $\eta$ :

$$
\begin{aligned}
& 0^{\eta}=0^{m} ; \quad 1^{\eta}=1^{m} ; \quad c^{\eta}=c^{m} ; \\
& +^{\eta}=+\left.^{m}\right|_{\eta} ; \quad \circ^{\eta}=\left.{ }^{m}\right|_{\eta} ; \quad \leq^{\eta}=\leq\left.^{m}\right|_{\eta} \quad(\text { restrições à } \eta) .
\end{aligned}
$$


De fato, notemos primeiramente que $0^{m}, 1^{m}, c^{m} \in \eta$, pois

$$
m \models 0^{m} \leq 0, m \models 1^{m} \leq 1 \quad \text { e } \quad m \models c^{m} \leq c .
$$

Basta agora mostrar que $+^{\eta}$ e $o^{\eta}$ são fechadas em $\eta$. Sejam então $a, b \in \eta$. Então existem termos $t_{1}(x)$ e $t_{2}(x)$ de $\mathcal{L}$ tais que

$$
m \vDash a \leq t_{1}(c) \quad \text { e } \quad m \vDash b \leq t_{2}(c) .
$$

Tomando o termo $t_{1}(x)+t_{2}(x)$, teremos então que $m \models a+b \leq t_{1}(c)+t_{2}(c)$ e, portanto, $a+b \in \eta$.

Analogamente, $a . b \in \eta$. Isso prova a Afirmação 3.

Afirmação 4: $\eta$ é subestrutura de $m$.

De fato, pela definição dada na Afirmação 3, temos que

$$
0^{\eta}=0^{m}, \quad 1^{\eta}=1^{m} \quad \text { e } \quad c^{\eta}=c^{m} .
$$

Também temos trivialmente que para todo $a, b \in \eta$,

$$
\begin{gathered}
a+{ }^{\eta} b=a+\left.{ }^{m}\right|_{\eta} b=a+{ }^{m} b \\
a_{\circ}{ }^{\eta} b=\left.a_{\circ}{ }^{m}\right|_{\eta} b=a_{\circ}{ }^{m} b \\
\mathrm{e} \quad a \leq^{\eta} b \Leftrightarrow a \leq\left.^{m}\right|_{\eta} b \Leftrightarrow a \leq^{m} b .
\end{gathered}
$$

Isso prova a Afirmação 4 .

Afirmação 5: Se $\varphi(x) \in \Sigma_{0}$, então para todo $a \in \eta$,

$$
m \models \varphi[a] \Leftrightarrow \eta \models \varphi[a] .
$$


De fato, mostremos por indução no comprimento de $\varphi$ :

- Se $\varphi$ é atômica, então para todo $a \in \eta$,

$$
m \vDash \varphi[a] \Leftrightarrow \eta \vDash \varphi[a]
$$

pela Afirmação 4 .

- Se $\varphi$ é $\neg \psi$, então para todo $a \in \eta$,

$$
\begin{aligned}
& m \models \varphi[a] \Leftrightarrow m \models \neg \psi[a] \Leftrightarrow m \forall \psi[a] \\
& \text { hip. indução } \eta \forall \psi[a] \Leftrightarrow \eta \models \neg \psi[a] \Leftrightarrow \eta \models \varphi[a] .
\end{aligned}
$$

- Se $\varphi$ é $\psi_{1} \wedge \psi_{2}, \psi_{1} \vee \psi_{2}$ ou $\psi_{1} \rightarrow \psi_{2}$, a prova é análoga ao caso anterior.

- $\operatorname{Se} \varphi$ é $\forall y \leq x \psi(y)$ e $a \in \eta$,

$$
\begin{aligned}
(\Rightarrow) & m \models \varphi[a] \Leftrightarrow m \models \forall y \leq a \psi(y) \\
& \Leftrightarrow \text { para todo } b \in m \operatorname{com} b \leq^{m} a: m \models \psi[b] \\
& \stackrel{\eta \subsetneq m}{\Rightarrow} \text { para todo } b \in \eta, \operatorname{com} b \leq^{\eta} a: m \models \psi[b] \\
& \text { Hip. indução para todo } b \in \eta, \operatorname{com} b \leq^{\eta} a: \eta \models \psi[b] \\
& \Leftrightarrow \eta \models \forall y \leq a \psi(y) \Leftrightarrow \eta \models \varphi[a] .
\end{aligned}
$$

$(\Leftarrow)$ Primeiramente, uma observação: se $a \in \eta$ e $b \in m, \operatorname{com} b \leq^{m} a$, então $b \in \eta$, pois como $a \in \eta$, então para algum termo $t(x)$ de $\mathcal{L}, m \models a \leq t(c)$ e, portanto, $m \models b \leq t(c)$, ou seja, $b \in \eta$.

Suponhamos então que $\eta \models \forall y \leq a \psi(y)$. Devemos mostrar que $m \models \forall y \leq$ $a \psi(y)$. Seja dado $b \in m$ tal que $b \leq^{m} a$. Como $a \in \eta$, pela observação acima, 
temos que $b \in \eta$ e, então, $b \leq \eta a$. Como $\eta \vDash \forall y \leq a \psi(y)$, temos então que $\eta \models \psi[b]$.

Como $b \in \eta$, por hipótese de indução temos que $m \models \psi[b]$. Portanto,

$$
m \vDash \forall y \leq a \psi(y) \text {, isto é, } m \models \varphi[a] .
$$

- Se $\varphi$ é $\exists y \leq x \psi(x)$, então para todo $a \in \eta$,

$(\Rightarrow)=\varphi[a] \Leftrightarrow m \vDash \exists y \leq a \psi(y) \Leftrightarrow$ existe $b \in m \operatorname{com} b \leq^{m} a: m \models \psi[b]$. Como $b \in m, a \in \eta$ e $b \leq^{m} a$, pela observação no item anterior, segue que $b \in \eta$, com $b \leq \eta a$. Portanto, existe $b \in \eta$, com $b \leq \eta a$. Portanto, existe $b \in \eta$ com $b \leq{ }^{\eta} a: m \models \psi[b]$. Por hipótese de indução, $\eta \models \psi[b]$. Então existe $b \in \eta$ com $b \leq{ }^{\eta} a: \eta \models \psi[b]$, ou seja, $\eta \models \exists y \leq a \psi(y)$, ou ainda, $\eta \models \varphi[a]$.

$(\Leftarrow) \eta \models \varphi[a] \Leftrightarrow \eta \vDash \exists y \leq a \psi(y) \Leftrightarrow$ existe $b \in \eta \operatorname{com} b \leq^{n} a: \eta \models \psi[b] \stackrel{\eta \subseteq m}{\Rightarrow}$ existe

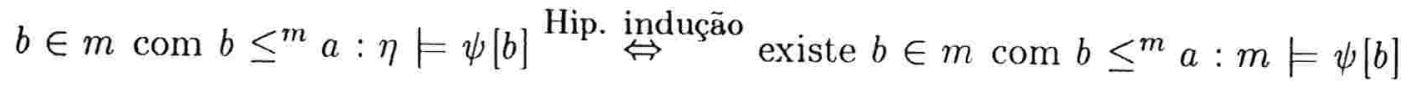
$\Leftrightarrow m \vDash \exists y \leq a \psi(y) \Leftrightarrow m \vDash \varphi[a]$.

Isso prova a Afirmação 5.

Afirmação 6: $\eta \models P^{-}$.

De fato, os axiomas 1-13 de $P^{-}$pertencem à $\Sigma_{0}$, portanto, pela Afirmação 5 , são satisfeitas em $\eta$. Resta mostrar que o Axioma 14, a saber,

$$
x<y \rightarrow \exists z(z+x+1=y)
$$

também é satisfeito em $\eta$. 
Sejam $a, b \in \eta$ tal que $\eta \vDash a<b$. Então, pela Afirmação $5, m \vDash a<b$. Como $m \models P^{-}$e, em particular, $m$ satisfaz o Axioma 14 , segue que $m \models \exists z(z+a+1=b)$. Seja $k \in m$ tal que $m \models k+a+1=b$. Decorre do Axioma 13 que $m \models k<k+a+1$. Portanto, $m \models k<b$. Mas $b \in \eta$, logo, existe um termo $t(x)$ de $\mathcal{L}$ tal que $m \models b<t(c)$ e, portanto, temos que $m \models k<t(c)$, ou seja, $k \in \eta$. Portanto, $\eta \models \exists z(z+a+1=b)$ e o Axioma 14 é verdadeiro em $\eta$.

Isso prova a Afirmação 6.

Afirmação 7: $\eta \models \forall y \neg \varphi(c, y)$.

De fato, suponha que $\eta \not \models \forall y \neg \varphi(c, y)$, ou seja, $\eta \models \exists y \varphi(c, y)$. Então existe $a \in \eta$ tal que $\eta \models \varphi(c, a)$. Como $a \in \eta$, existe um termo $t(x)$ de $\mathcal{L}$ tal que $m \vDash a \leq t(c)$.

Segue da Afirmação 5 que $\eta \vDash a \leq t(c)$. Portanto, existe um termo $t(x)$ de $\mathcal{L}$ tal que $\eta \models \exists y \leq t(c) \varphi(c, y)$.

Como $\varphi \in \Sigma_{0}$, então $\exists y \leq t(c) \varphi(c, y) \in \Sigma_{0}$, então pela Afirmação 5 , temos que

$$
m \vDash \exists y \leq t(c) \varphi(c, y)
$$

Por outro lado, da Afirmação 2, temos que

$$
m \models \forall y \leq t(c) \neg \varphi(c, y)
$$

ou seja, $m \not \models \exists y \leq t(c) \varphi(c, y)$, uma contradição.

Isso prova a Afirmação 7 .

Afirmação 8: $\eta \models I \Sigma_{0}$. 
De fato, consideremos o conjunto

$$
\Gamma=\left\{\forall x[\varphi(0) \wedge \forall y<x(\varphi(y) \rightarrow \varphi(y+1)) \rightarrow \varphi(x)]: \varphi \in \Sigma_{0}\right\}
$$

Mostremos primeiramente que $\eta \models \Gamma$.

Seja $\psi(x)$ a seguinte fórmula:

$$
\varphi(0) \wedge \forall y<x(\varphi(y) \rightarrow \varphi(y+1)) \rightarrow \varphi(x)
$$

onde $\varphi \in \Sigma_{0}$. Devemos mostrar que $\eta \models \forall x \psi(x)$.

Seja $a \in \eta$. Como $m \models\left(P^{-}+I \Sigma_{0}\right)^{\prime}$. Então, $m \models P^{-}+I \Delta_{0}$ e, pelo Lema $9.19, m \models \Gamma$. Logo, $m \models \forall x \psi(x)$ e como $\eta \subseteq m$, então $a \in m$ e, portanto, $m \vDash \psi[a]$. Observando que $\psi \in \Sigma_{0}$ e $a \in \eta$, pela Afirmação 5, segue que $\eta \models \psi[a]$. Então, $\eta \models \forall x \psi(x)$ e isso mostra que $\eta \models \Gamma$. Também, pela Afirmação $6, \eta \models P^{-}$. Portanto, pelo Lema 9.19 , segue que $\eta \models I \Sigma_{0}$.

Isso prova a Afirmação 8.

Podemos agora finalizar a demonstração do Teorema 9.20. A hipótese do Teorema de Parikh é

$$
P^{-}+I \Sigma_{0} \vdash \forall x \exists y \varphi(x, y)
$$

donde $\left(P^{-}+I \Sigma_{0}\right)^{\prime} \vdash \exists y \varphi(c, y)$.

Pelas Afirmações 6 e 8 , temos que $\eta \models P^{-}+I \Sigma_{0}$. Logo, $\eta \models\left(P^{-}+I \Delta_{0}\right)^{\prime}$ e, portanto, $\eta \vDash \exists y \varphi(c, y)$. 
Por outro lado, pela Afirmação 7, temos que

$$
\eta \vDash \forall y \neg \varphi(c, y)
$$

ou seja,

$$
\eta \not \models \exists y \varphi(c, y)
$$

uma contradição. Portanto, existe um termo $t(x)$ de $\mathcal{L}$ tal que

$$
P^{-}+I \Sigma_{0} \vdash \forall x \exists y \leq t(x) \varphi(x, y)
$$

\subsection{O teorema de Wilkie}

Lema 9.21 Seja $L \subseteq \Sigma^{*}$ e $f: \Sigma^{*} \rightarrow \Sigma^{*}$ uma funçâo computável em tempo polinomial, $e$ seja

$$
Q=\left\{x \in \Sigma^{*}: f(x) \in L\right\}
$$

Então,

a) $L \in \mathbf{P} \Rightarrow Q \in \mathbf{P}$;

b) $L \in \mathbf{N P} \Rightarrow Q \in \mathbf{N P}$.

Demonstração: Seja $T_{f}$ uma DTM que calcula $f$ e $p(x)$ um polinômio tal que $\forall x \in \Sigma^{*}$ : $\theta_{T_{f}}(x) \leq p(|x|)$

a) Seja $T$ uma DTM que aceita $L$ e $q(x)$ um polinômio tal que se $x \in L$, então $\theta_{T}(x) \leq q(|x|)$. 
Observando que uma DTM não pode imprimir mais símbolos que o número total de passos na computação, temos que

$$
|f(x)| \leq|x|+p(|x|)
$$

(a presença do termo $|x|$ é devida à entrada $x$ já impressa na fita).

Se $s(n)=n$, podemos ainda escrever

$$
|f(x)| \leq s(|x|)+p(|x|) .
$$

E lembrando que os polinômios considerados têm coeficientes positivos, temos então que

$$
\theta_{T}(f(x)) \leq q(|f(x)|) \leq q(r(|x|)+p(|x|))
$$

Agora uma DTM $R$ que aceita $Q=\left\{x \in \Sigma^{*}: f(x) \in L\right\}$ pode ser facilmente construída: para $x \in \Sigma^{*}$, por meio da DTM $T_{f}$ calculamos $f(x) \operatorname{com} \theta_{T_{f}}(x) \leq p(|x|)$.

Depois utilizamos a DTM $T$ para determinar se $f(x) \in L$ com

$$
\theta_{T}(f(x)) \stackrel{(*)}{\leq} q(r(|x|)+p(|x|))
$$

Então, a DTM $R$ aceita $Q$, com

$$
\theta_{R}(x) \leq p(|x|)+q(r(|x|)+p(|x|))
$$

Portanto, $Q \in \mathbf{P}$.

b) A demonstração é análoga à parte a), substituindo a DTM que aceita $L$ por uma NDTM que aceita $L$. 
Lema 9.22 Se existe uma linguagem $L \subseteq \Sigma^{*}$ tal que $L$ é coNP-completo e $L \in \mathbf{N P}$, entäo NP $=$ coNP.

Demonstração: Notação: se $S \in \Sigma^{*}, S^{c}=\Sigma^{*} \backslash S$.

- Mostremos que NP $\subseteq$ coNP.

Seja $S \in \mathrm{NP}$. Então, $S^{c} \in \operatorname{coNP}$ e como $L$ é coNP-completo, temos que $S^{c} \leq_{p} L$, ou seja, existe uma função $f: \Sigma^{*} \rightarrow \Sigma^{*}$ computável em tempo polinomial tal que

$$
x \in S^{c} \leftrightarrow f(x) \in L
$$

Seja $Q=\left\{x \in \Sigma^{*}: f(x) \in L\right\}$. Como por hipótese $L \in \mathrm{NP}$, pelo Lema 9.21 , temos que $Q \in$ NP. Então,

$$
x \in Q \leftrightarrow f(x) \in L \leftrightarrow x \in S^{c} .
$$

Portanto, $Q=S^{c}$, e como $Q \in \mathrm{NP}$, então $S^{c} \in \mathrm{NP}$, logo, $S \in$ coNP. Isso mostra que NP $\subseteq$ coNP.

- Mostremos agora que coNP $\subseteq$ NP.

Seja $S \in$ coNP. Como $L$ é coNP-completo, então $S \leq_{p} L$, ou seja, existe uma função $f: \Sigma^{*} \rightarrow \Sigma^{*}$ computável em tempo polinomial tal que

$$
x \in S \leftrightarrow f(x) \in L
$$


Seja $Q=\left\{x \in \Sigma^{*}: f(x) \in L\right\}$. Como por hipótese $L \in \mathrm{NP}$, pelo Lema 9.21 , temos que $Q \in$ NP. Então,

$$
x \in Q \leftrightarrow f(x) \in L \leftrightarrow x \in S .
$$

Portanto, $Q=S$ e como $Q \in \mathrm{NP}$, então $S \in \mathrm{NP}$.

Isso mostra que coNP $\subseteq$ NP. Portanto, $\mathbf{N P}=$ coNP.

Lema 9.23 Uma linguagem $L$ é NP-completa se e somente se $L^{c}$ é coNP-completa.

Demonstração: $(\Rightarrow)$ Suponhamos que $L$ é NP-completa. Seja $Q \in$ coNP. Então $Q^{c} \in \mathrm{NP}$ e $Q^{c} \leq_{p} L$, ou seja, existe uma função $f$ computável em tempo polinomial tal que $x \in Q^{c} \leftrightarrow f(x) \in L$, ou seja, $x \in Q \leftrightarrow f(x) \in L^{c}$. Portanto, $L^{c} \in$ coNP e para toda linguagem $Q \in \operatorname{coNP}, Q \leq{ }_{p} L^{c}$, isto é, $L^{c}$ é coNP-completa.

A recíproca é mostrada de forma análoga.

Lema 9.24 Seja $t(x, y, z)$ um termo de $\mathcal{L}$ e $P(x, y, z, \vec{z})$ e $Q(x, y, z, \vec{z})$ polinômios com. coeficientes positivos. Então o problema.

$$
S=\left\{(a, b, c) \in \mathbb{N}^{3}: \exists \vec{z} \leq t(a, b, c) P(a, b, c, \vec{z})=Q(a, b, c, \vec{z})\right\}
$$

é NP.

Demonstração: (Esboço) 
Codificando convenientemente $n$-uplas de números naturais, por exemplo em linguagem binária, e lembrando que uma linguagem $L \in \mathrm{NP}$ se e somente se existem um polinômio $p(x)$ e uma linguagem $L^{\prime} \in \mathbf{P}$ tal que

$$
x \in L \leftrightarrow \exists y\left(|y| \leq p(|x|) \wedge(x, y) \in L^{\prime}\right)
$$

podemos ver que o problema $S \in \mathrm{NP}$, já que o limitante $t(x, y, z)$ é um termo de $\mathcal{L} \mathrm{e}$, portanto, um polinômio. Além disso, o cálculo de um polinômio, bem como a verificação de uma igualdade podem ser feitas em tempo polinomial

$$
\text { Portanto, } S \in \mathbf{N P} \text {. }
$$

Temos agora os pré-requisitos para enunciar e esboçar uma prova do principal resultado deste capítulo.

O cerne do MRDP consiste em mostrar que toda fórmula $\varphi \in \Sigma_{1}$ é equivalente a uma fórmula diofantina. Aqui, provar o MRDP em $I \Sigma_{0}$ significa que para toda fórmula $\varphi \in \Sigma_{1}$ existe uma fórmula diofantina $\psi$, tal que

$$
P^{-}+I \Sigma_{0} \vdash \varphi \leftrightarrow \psi
$$

Para provar o teorema de Wilkie, vamos usar um resultado obtido por Manders e Adleman que apresentamos a seguir, omitindo a demonstração, que pode ser encontrada em [7].

Teorema 9.25 (Manders-Adleman) O problema

$$
\left\{(a, b, c) \in \mathbb{N}^{3}: \exists x \leq c \exists y \leq c\left(a x^{2}+b y=c\right)\right\}
$$

é NP-completo. 
Teorema 9.26 (Wilkie) Se o MRDP puder ser provado em $I \Sigma_{0}$, então $\mathrm{NP}=$ coNP.

Demonstração: A fórmula $\forall x \leq c \forall y \leq c\left(a x^{2}+b y \neq c\right) \in \Sigma_{1}$. Se o MRDP puder ser provado em $I \Sigma_{0}$, então existem polinômios $P(x, y, z, \vec{w})$ e $Q(x, y, z, \vec{w})$ tais que

$$
P^{-}+I \Sigma_{0} \vdash \forall a, b, c\left[\forall x \leq c \forall y \leq c\left(a x^{2}+b y \neq c\right) \leftrightarrow \exists \vec{w}(P(a, b, c, \vec{w})=Q(a, b, c, \vec{w}))\right],
$$

de onde obtemos (daqui para frente, vamos omitir as variáveis $a, b, c, \vec{w}$ dos polinômios $P(a, b, c, \vec{w})$ e $Q(a, b, c, \vec{w})):$

$$
P^{-}+I \Sigma_{0} \vdash \forall a, b, c\left[\forall x \leq c \forall y \leq c\left(a x^{2}+b y \neq c\right) \rightarrow \exists \vec{w}(P=Q)\right]
$$

$\mathrm{e}$

$$
P^{-}+I \Sigma_{0} \vdash \forall a, b, c\left[\exists \vec{w}(P=Q) \rightarrow \forall x \leq c \forall y \leq c\left(a x^{2}+b y \neq c\right)\right]
$$

Vamos analisar cada uma das implicações:

- Se $P^{-}+I \Sigma_{0} \vdash \forall a, b, c\left[\forall x \leq c \forall y \leq c\left(a x^{2}+b y \neq c\right) \rightarrow \exists \vec{w}(P=Q)\right]$.

Tomando $\vec{w}$ distintas de $a, b, c$, obtemos

$$
P^{-}+I \Sigma_{0} \vdash \forall a, b, c \exists \vec{w}\left[\forall x \leq c \forall y \leq c\left(a x^{2}+b y \neq c\right) \rightarrow P=Q\right] .
$$

Como $\forall x \leq c \forall y \leq c\left(a x^{2}+b y \neq c\right) \rightarrow P=Q$ é uma $\Sigma_{0}$-fórmula, pelo Teorema de Parikh existe um termo $t(a, b, c)$ de $\mathcal{L}$ tal que

$$
P^{-}+I \Sigma_{0} \vdash \forall a, b, c \exists \vec{w} \leq t(a, b, c)\left[\forall x \leq c \forall y \leq c\left(a x^{2}+b y \neq c\right) \rightarrow P=Q\right]
$$

isso implica

$$
P^{-}+I \Sigma_{0} \vdash \forall a, b, c\left[\forall x \leq c \forall y \leq c\left(a x^{2}+b y \neq c\right) \rightarrow \exists \vec{w} \leq t(a, b, c)(P=Q)\right] .
$$

Por outro lado, 
- se $P^{-}+I \Sigma_{0} \vdash \forall a, b, c\left[\exists \vec{w}(P=Q) \rightarrow \forall x \leq c \forall y \leq c\left(a x^{2}+b y \neq c\right)\right]$. Como

$$
P^{-}+I \Sigma_{0} \vdash \exists \vec{w} \leq t(a, b, c)(P=Q) \rightarrow \exists \vec{w}(P=Q),
$$

teremos que

$$
P^{-}+I \Sigma_{0} \vdash \forall a, b, c\left[\exists \vec{w} \leq t(a, b, c)(P=Q) \rightarrow \forall x \leq c \forall y \leq c\left(a x^{2}+b y \neq c\right)\right] .
$$

Mostramos portanto que

$$
P^{-}+I \Sigma_{0} \vdash \forall a, b, c\left[\forall x \leq c \forall y \leq c\left(a x^{2}+b y \neq c\right) \leftrightarrow \exists \vec{u} \leq t(a, b, c)(P=Q)\right] .
$$

Consideremos agora os seguintes conjuntos:

$$
\begin{aligned}
R & =\left\{(a, b, c) \in \mathbb{N}^{3}: \forall x \leq c \forall y \leq c\left(a x^{2}+b y \neq c\right)\right\} \quad \mathrm{e} \\
S & =\left\{(a, b, c) \in \mathbb{N}^{3}: \exists \vec{w} \leq t(a, b, c)(P=Q)\right\} .
\end{aligned}
$$

Notemos então que pelo Teorema de Manders-Adleman o conjunto $R^{c}$ é NP-completo e, portanto, pelo Lema 9.23, $R$ é coNP-completo, e pelo Lema 9.24, $S \in \mathrm{NP}$.

Por outro lado, como $\mathbb{N} \models P^{-}+I \Sigma_{0}$, então

$$
\mathbb{N} \models \forall a, b, c\left[\forall x \leq c \forall y \leq c\left(a x^{2}+b y \neq c\right) \leftrightarrow \exists \vec{w} \leq t(a, b, c)(P=Q)\right],
$$

e isso mostra que $R=S$.

Portanto, $R \in \mathrm{NP}$ e é coNP-completo. Pelo Lema 9.22, temos então que

$$
\mathrm{NP}=\operatorname{coNP}
$$




\section{Referências bibliográficas}

[1] M. Davis. Hilbert's tenth problem is insolvable. Am. Math. Monthly. (March 1973), 233-269.

[2] M. Davis, H. Putnam, J. Robinson. The decision problem for exponential diophantine equations. Ann. Math., 74 (1961), 425-436.

[3] H. Gaifman, C. Dimitracopoulos. Fragments of Peano's arithmetic and the MRDP theorem. Collection: Logic and Algorithmic, Zurich (1980), 187-206.

[4] G.H. Hardy and E. Wright. An Introduction to the Theory of Numbers. 4.ed. Oxford University Press (1960).

[5] J.P. Jones, Y. Matyasevic. Proof of recursive unsolvability of Hilbert's tenth problem. Am. Math. Monthly, 98(8) (1991), 689-709.

[6] S.C. KLEenE. Introduction to Metamathematics. Van Nostrand, New York (1952).

[7] K.L. Manders, L. Adleman. NP-complete decision problems for binary quadratics. J. Comp. Syst. Sci., 16 (1978), 168-184.

[8] Y.V. Matyasevic. Enumerable sets are diophantine. Doklady Akademii Nauk, SSSR, 191 (1970), 279-282.

[9] Yuri Matyasevic. Diophantine representation of enumerable predicates (Russian). Izv. Akad. Nauk SSSR, Ser. Mat., 35 (1971), 3-30.

[10] R. PARIKh. Existence of feasibility in arithmetic. J. Symb. Logic, 36 (1971), 494-508.

[11] Júlia Robinson. Existencial definability in arithmetic. Trans. Amer. Math. Soc., 72 (1952), 437-449. 
[12] H. Rogers. Theory of Recursive Functions and Effective Computability. McGrawHill, New York (1967).

[13] A.J. WilkiE. Applications of complexity theory to $\Sigma_{0}$-definibility problems in arithmetic. In: Pacholski et al., eds., Model Theory, Algebra and Arithmetic, Proc. Karpacz, Poland (1979). Lect. Notes in Math., 834, Springer, Berlin (1980), 363369. 